\title{
ON STATICALLY ADMISSIBLE STRESS FIELDS FOR A PLANE MASONRY-LIKE STRUCTURE
}

\author{
BY \\ MAURIZIO ANGELILLO (Istituto di Ingegneria Civile, Università di Salerno, Italy) \\ AND \\ FABIO ROSSO (Dipartimento di Matematica "Ulisse Dini", Università di Firenze, Italy)
}

SumMaRY. Explicit and directly verifiable nonlocal conditions for the existence of a statically admissible stress field in an ideal plane body that does not support tension in the absence of body forces are presented. These conditions are sufficient for nonconvex bodies and become necessary and sufficient restricting to convex bodies and to loads different from zero at any point of the boundary.

1. Introduction. Masonry structures exhibit a sharp difference in resistance if compressed or pulled. As a first approach to a masonry-like material, an ideal "no-tension body" has been suggested: it reacts elastically to arbitrary pressures but cannot bear the slightest traction. This model has been the object of several investigations in recent years (see, for example, [1], [2], [3], [5] and references therein); among these Giaquinta and Giusti's work [3] is the one of more interest to us and has mainly motivated the present research.

Here we focus our attention on one particular aspect of the theory which is purely static: an equilibrated and purely compressive stress field may not necessarily exist corresponding to arbitrarily given surface loads $\mathbf{p}$ and body forces $\mathbf{b}$. In this note we present explicit conditions for the compatibility of the boundary data. These conditions are nonlocal, being given in terms of the resultant $\mathbf{R}$ and the moment $\mathbf{M}$ of the loads on portions of the boundary and turn out to be directly verifiable; actually they only involve the study of the sign of a given real-valued function of two variables. Moreover, these conditions are sufficient for arbitrary plane bodies and also necessary for convex ones, if one restricts to loads $\mathbf{p}$ different from zero at any point of the boundary.

This result represents an extension of a similar one given in [3] (Secs. 7 and 8).

In [3] the authors, under the assumption specified below, prove the existence of a weak solution of the boundary-value problem for a plane body $\Omega$ composed of normal elastic no-tension material (in the sense of Del Piero [2]). These assumptions

Received April 26, 1993.

1991 Mathematics Subject Classification. Primary 73B99, 73C99, 58E30.

(C)1995 Brown University 
are

a) $\mathbf{p} \in L^{\infty}(\partial \Omega)$,

b) $\mathbf{b} \in L^{2}(\Omega)$,

c) $\Omega$ open, bounded and connected with Lipschitz boundary. Moreover, they assume that

d) $(\mathbf{p}, \mathbf{b})$ is a "safe" load,

that is, there exists a stress field, equilibrated with $\mathbf{p}$ and $\mathbf{b}$ whose eigenvalues are bounded away from zero by some negative constant.

In Sec. 8 of [3] Giaquinta and Giusti give explicit conditions on the load for (d) to hold. Their result is obtained under the following hypotheses:

(i) $\Omega$ convex and bounded,

(ii) $\partial \Omega$ of class $C^{\prime}$,

(iii) $\mathbf{b}=0$,

(iv) $\mathbf{p} \in C^{1}(\partial \Omega)$,

(v) $\mathbf{p}(x) \neq 0, \forall \mathbf{x} \in \partial \Omega$,

(vi) $\mathbf{R} \neq 0, \mathbf{M} \neq 0$ on $\partial \Omega \times \partial \Omega$.

The conditions they give are sufficient for the existence of an equilibrated and purely compressive stress field.

The conditions we present in this paper are proved under the sole hypotheses (iii), $(\mathrm{v})$, and

(i) ${ }^{*} \bar{\Omega}$ simply connected and bounded,

(ii) ${ }^{*} \partial \Omega$ of class $C^{2}$,

(iv) ${ }^{*} \mathbf{p} \in C^{2}(\partial \Omega)$,

and turn out to be also necessary under the further assumption (i). Notice that assumption (vi) is no longer needed in the present paper; therefore, the resultant $\mathbf{R}$ and the moment $\mathbf{M}$ are allowed to be zero on portions of the boundary, that is, we include loads that are admissible but not safe. Covering the complete class of admissible loads (safe and limit) would require dropping assumption ( $v$ ).

The problem is mathematically formulated in Sec. 2. Section 3 contains the proof of the Main Theorem stated in Sec. 2. Appendix I is devoted to some minor proofs. The hypothesis (v) plays a crucial role throughout the proof; in Appendix II our result is extended to some, more general, loading conditions.

2. Mathematical setup. Let $\Omega$ be the plane, bounded, and simply connected region occupied by a two-dimensional no-tension body $\mathscr{B}$. We remark explicitly that $\Omega$ may be either convex or nonconvex. The boundary $\partial \Omega$ of $\Omega$ is supposed to be of class $C^{2}$; we represent $\partial \Omega$ parametrically by $\mathbf{x}=\mathbf{x}(s), s \in[0, L)$ with $L<\infty$, and where $s$ measures the arclength along $\partial \Omega$.

Let $\partial \Omega$ be positively oriented in the direction of its unit tangent vector field $\mathbf{t}(s)=\frac{d \mathbf{x}}{d s}$. We suppose the plane containing $\Omega$ to be positively oriented in the direction of the unit vector e defined by

$$
\mathbf{e}=\mathbf{n}(s) \times \mathbf{t}(s),
$$

$\mathbf{n}(s)$ being the outward unit normal along $\partial \Omega$. 


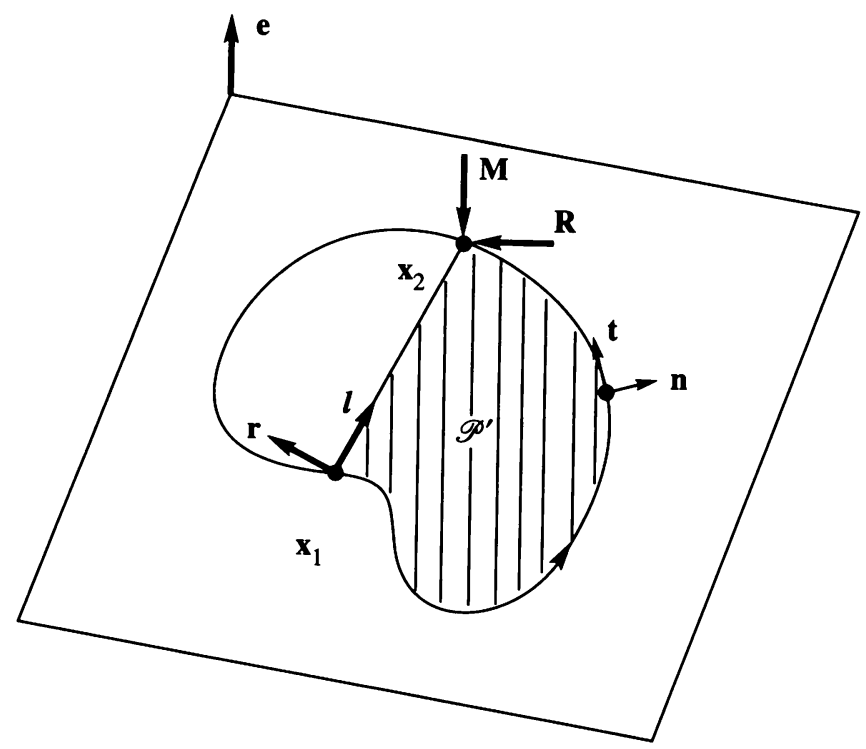

FIG. 1.

In the absence of body forces, a stress field $\mathbf{T}$ over $\Omega$ is said to be equilibrated with the load $\mathbf{p}$ on $\partial \Omega$ if $\mathbf{T}$ satisfies the set of equations

$$
\begin{cases}\boldsymbol{\nabla} \cdot \mathbf{T}=0, & \text { in } \Omega, \\ \mathbf{T}=\mathbf{T}^{\mathbf{T}}, & \text { in } \Omega, \\ \mathbf{T} \cdot \mathbf{n}=\mathbf{p}, & \text { on } \partial \boldsymbol{\Omega},\end{cases}
$$

where $\mathbf{p}$ is given with the regularity $\mathbf{p} \in C^{2}(\partial \Omega)$. Our subsequent analysis is restricted to the case $\mathbf{p} \neq 0$ everywhere along $\partial \Omega$; the more general case $\mathbf{p}=0$ on some portions of $\partial \Omega$ will be considered in Appendix II, for some special cases.

We shall refer to a tensor field $\mathbf{T}$ of class $C^{1}(\bar{\Omega})$, such that $\boldsymbol{\nabla} \cdot \mathbf{T}$ is meaningful in the ordinary sense and which solves (1), as a classical solution.

Since $\mathscr{B}$ does not react to tensile loads, the following constraints on the invariants of $\mathbf{T}$ need to be added to (1):

$$
\left\{\begin{array}{l}
\operatorname{tr} \mathbf{T} \leq 0 \\
\operatorname{det} \mathbf{T} \geq 0 .
\end{array}\right.
$$

We call $\mathbf{T}$ a statically admissible stress field (s.a.f.) for the load $\mathbf{p}$ if $\mathbf{T}$ is a classical solution of (1) that satisfies (2).

Evidently a solution to (1)-(2) does not generally exist if $\mathbf{p}$ is arbitrarily given. Indeed $\mathbf{p}$ has to be at least balanced, (1) being a traction problem. Moreover, because of the constraint (2) on $\mathbf{T}$, one should expect that further compatibility conditions be imposed on the load $\mathbf{p}$ in order for a statically admissible stress field to exist. The definition of these compatibility conditions is precisely the scope of the present paper; to discuss them we need to introduce some definitions.

Let $\mathbf{x}_{1}$ and $\mathbf{x}_{2}$ be any two points of $\partial \Omega$ and let us denote by $\gamma\left(\mathbf{x}_{1}, \mathbf{x}_{2}\right)$ the portion of $\partial \Omega$ connecting $\mathbf{x}_{1}=\mathbf{x}\left(s_{1}\right)$ with $\mathbf{x}_{2}=\mathbf{x}\left(s_{2}\right)$, following the positive direction along 
$\partial \Omega$. Let also

$$
\mathbf{R}\left(\mathbf{x}_{1}, \mathbf{x}_{2}\right) \equiv \int_{\gamma\left(\mathbf{x}_{1}, \mathbf{x}_{2}\right)} \mathbf{p}(\mathbf{x})
$$

be the resultant of the surface tractions on $\gamma\left(\mathbf{x}_{1}, \mathbf{x}_{2}\right)$. Similarly let

$$
\mathbf{M}\left(\mathbf{x}_{1}, \mathbf{x}_{2}\right) \equiv \int_{\gamma\left(\mathbf{x}_{1}, \mathbf{x}_{2}\right)} \mathbf{p}(\mathbf{x}) \times\left(\mathbf{x}_{2}-\mathbf{x}\right)
$$

be the moment of $\mathbf{p}$ along $\gamma\left(\mathbf{x}_{1}, \mathbf{x}_{2}\right)$ with respect to $\mathbf{x}_{2}$. If $\mathbf{x}_{1}=\mathbf{x}(0)$ and $\mathbf{x}_{2}=\mathbf{x}(s)$ we shall write

$$
\widehat{\mathbf{R}}(s) \equiv \mathbf{R}(\mathbf{x}(0), \mathbf{x}(s)), \quad \widehat{\mathbf{M}}(s) \equiv \mathbf{M}(\mathbf{x}(0), \mathbf{x}(s)) .
$$

We denote by $\mathbf{r}\left(\mathbf{x}_{1}, \mathbf{x}_{2}\right)$ the unit vector

$$
\mathbf{r}\left(\mathbf{x}_{1}, \mathbf{x}_{2}\right)=\mathbf{e} \times \ell\left(\mathbf{x}_{1}, \mathbf{x}_{2}\right)
$$

with $\ell\left(\mathbf{x}_{1}, \mathbf{x}_{2}\right)=\left|\mathbf{x}_{2}-\mathbf{x}_{1}\right|^{-1}\left(\mathbf{x}_{2}-\mathbf{x}_{1}\right)$. Let also $\operatorname{seg}\left(\mathbf{x}_{1}, \mathbf{x}_{2}\right)$ be the set

$$
\operatorname{seg}\left(\mathbf{x}_{1}, \mathbf{x}_{2}\right) \equiv\left\{\mathbf{x} \in \mathbf{R}^{2} \mid \mathbf{x}=\mathbf{x}_{1}+\lambda\left(\mathbf{x}_{2}-\mathbf{x}_{1}\right), \lambda \in(0,1)\right\},
$$

and, setting $\mathscr{H} \equiv \partial \Omega \times \partial \Omega$, let us finally denote by $\mathscr{H}_{\text {in }}$ the set

$$
\mathscr{H}_{\text {in }} \equiv\left\{\left(\mathbf{x}_{1}, \mathbf{x}_{2}\right) \in \mathscr{H} \mid \operatorname{seg}\left(\mathbf{x}_{1}, \mathbf{x}_{2}\right) \subset \Omega\right\} .
$$

Throughout the present paper $\partial \Omega$ (and consequently $\mathscr{H}$ ) is thought of as endowed with the relative topology induced by $\mathbf{R}^{2}$ onto $\partial \Omega$.

If $\left(\mathbf{x}_{1}, \mathbf{x}_{2}\right) \in \mathscr{H}_{\text {in }}, \operatorname{seg}\left(\mathbf{x}_{1}, \mathbf{x}_{2}\right)$ is called a section of $\Omega$ since $\Omega$ is split by $\operatorname{seg}\left(\mathbf{x}_{1}, \mathbf{x}_{2}\right)$ into two parts, $\mathscr{P}^{\prime}$ and $\mathscr{P}^{\prime \prime}$; we identify $\mathscr{P}^{\prime}$ as the region such that

$$
\partial \mathscr{P}^{\prime}=\gamma\left(\mathbf{x}_{1}, \mathbf{x}_{2}\right) \cup \overline{\operatorname{seg}}\left(\mathbf{x}_{1}, \mathbf{x}_{2}\right) \text {. }
$$

We also define

$$
\Gamma_{0} \equiv\{\mathbf{x} \in \partial \Omega \mid \mathbf{p}(\mathbf{x})=0\}, \quad \Gamma_{1} \equiv \partial \Omega-\Gamma_{0} .
$$

Since $\mathbf{p}(\mathbf{x})$ is continuous on $\partial \Omega, \Gamma_{0}$ is closed and $\Gamma_{1}$ is open with respect to the relative topology of $\partial \Omega$.

Proposition 1. Suppose that there exists a classical solution $\mathbf{T}$ to problem (1)-(2). Then, for any $\left(\mathbf{x}_{1}, \mathbf{x}_{2}\right) \in \mathscr{H}_{\text {in }}$, either

$$
\mathbf{R}\left(\mathbf{x}_{1}, \mathbf{x}_{2}\right)=\mathbf{M}\left(\mathbf{x}_{1}, \mathbf{x}_{2}\right)=0
$$

or

$$
\mathbf{M}\left(\mathbf{x}_{1}, \mathbf{x}_{2}\right) \cdot \mathbf{e}<0
$$

Moreover,

$$
\mathbf{n}(\mathbf{x}) \cdot \mathbf{p}(\mathbf{x})<0, \quad \forall \mathbf{x} \in \Gamma_{1} .
$$

Let us now define

$$
\begin{aligned}
& \mathscr{H}_{1} \equiv\left\{\left(\mathbf{x}_{1}, \mathbf{x}_{2}\right) \in \mathscr{H} \mid(\text { A } 1) \text { holds }\right\}, \\
& \mathscr{H}_{2} \equiv\left\{\left(\mathbf{x}_{1}, \mathbf{x}_{2}\right) \in \mathscr{H} \mid \text { (A2) holds }\right\} .
\end{aligned}
$$

Since $\mathbf{R}$ and $\mathbf{M}$ are continuous over $\mathscr{H}, \mathscr{H}_{1}$ is closed while, if $\mathscr{H}=\mathscr{H}_{1} \cup \mathscr{H}_{2}, \mathscr{H}_{2}$ is open with respect to the relative topology of $\mathscr{H}$. 
RemarK 1. Using the classical formula $\mathbf{M}\left(\mathbf{x}_{1}, \mathbf{x}_{2}\right)=-\mathbf{M}\left(\mathbf{x}_{2}, \mathbf{x}_{1}\right)+\mathbf{R}\left(\mathbf{x}_{1}, \mathbf{x}_{2}\right) \times$ $\left(\mathbf{x}_{2}-\mathbf{x}_{1}\right)$, it is immediately verified that condition (A2), applied first to $\left(\mathbf{x}_{1}, \mathbf{x}_{2}\right)$ and then to $\left(\mathbf{x}_{2}, \mathbf{x}_{1}\right)$, implies

$$
\mathbf{R}\left(\mathbf{x}_{1}, \mathbf{x}_{2}\right) \cdot \mathbf{r}\left(\mathbf{x}_{1}, \mathbf{x}_{2}\right)>0, \quad \forall\left(\mathbf{x}_{1}, \mathbf{x}_{2}\right) \in \mathscr{H}_{2} .
$$

Propositions (A1), (A2), and (A2) have the following physical meaning: if a s.a.f. T does exist, then the corresponding action of $\mathscr{P}^{\prime}$ over $\mathscr{P}^{\prime \prime}$, if not equivalent to zero, consists of a compressive resultant whose central axis intersects the section $\operatorname{seg}\left(\mathbf{x}_{1}, \mathbf{x}_{2}\right)$. Finally, (A3) is an obvious consequence of (2).

The alternative (A1), (A2) was already shown by Del Piero [2] in a more general context.

Although (A1), (A2), and (A3) are necessary to the existence and their sufficiency seems very likely too, we are not able to prove it in full generality. We shall prove the existence of a classical solution $\mathbf{T}$ to (1)-(2) by assuming that either (A1) or (A2) be verified (for nonconvex bodies) on a larger set of arcs $\gamma\left(\mathbf{x}_{1}, \mathbf{x}_{2}\right)$, that is, for every $\left(\mathbf{x}_{1}, \mathbf{x}_{2}\right) \in \mathscr{H}$. Clearly this makes the set of admissible tractions smaller.

MAIN TheOREM. Let $\mathbf{p} \in C^{2}(\partial \Omega)$ be given in such a way that

(i) $\mathscr{H}=\mathscr{H}_{1} \cup \mathscr{H}_{2}$,

(ii) $\mathbf{p}(\mathbf{x}) \cdot \mathbf{n}(\mathbf{x})<0, \quad \forall \mathbf{x} \in \partial \Omega$,

(iii) for every $\left(s_{1}^{0}, s_{2}^{0}\right)$ with $s_{1}^{0} \neq s_{2}^{0}$ there exists $\beta\left(s_{1}^{0}, s_{2}^{0}\right)$ such that

$$
-\mathbf{M}\left[\mathbf{x}\left(s_{1}\right), \mathbf{x}\left(s_{2}\right)\right] \cdot \mathbf{e} \geq \beta\left[\left(s_{1}-s_{1}^{0}\right)^{2}+\left(s_{2}-s_{2}^{0}\right)^{2}\right]
$$

for every $\left(s_{1}, s_{2}\right) \in I_{1, \delta} \times I_{2, \delta}$ such that $\left(\mathbf{x}\left(s_{1}\right), \mathbf{x}\left(s_{2}\right)\right) \in \mathscr{H}_{2}$, where $I_{i, \delta}=$ $\left(s_{i}^{0}-\delta, s_{i}^{0}+\delta\right)$ and $\delta$ is a positive number such that $I_{1, \delta} \cap I_{2, \delta}=\varnothing$.

Then there exists a classical solution $\mathbf{T}$ to problem (1)-(2).

Throughout the sequel of the paper we shall need to have the problem written in terms of the Airy function.

With respect to a given system of Cartesian coordinates $\left(x_{1}, x_{2}\right)$, the Airy function's solution is defined through the equations

$$
T_{11}=\phi_{, 22}, \quad T_{12}=-\phi_{, 21}, \quad T_{22}=\phi_{, 11},
$$

where $\phi_{, i j}$ is a shorthand for $\frac{\partial^{2} \phi}{\partial x_{i} \partial x_{j}}$.

Constraints (2) are equivalent to requiring that $z=\phi(\mathbf{x})$ be a concave function on $\Omega$. Indeed, by substituting (7) in (2) we obtain the condition that the Hessian matrix of $\phi$ be negative semidefinite in $\Omega$, and this is equivalent to the concavity of the restriction of $\phi$ to each line segment in $\Omega$.

The boundary condition $(1)_{3}$ transforms (see [4, p. 158] for details) into

$$
\begin{gathered}
\phi(\mathbf{x}(s))=f(s), \\
\boldsymbol{\nabla} \phi(\mathbf{x}(s))=\mathbf{v}(s),
\end{gathered}
$$

where, recalling definitions (3), (4), and (5),

$$
\begin{aligned}
f(s) & \equiv \widehat{\mathbf{M}}(s) \cdot \mathbf{e}, \\
\mathbf{v}(s) & \equiv g(s) \mathbf{n}(s)+\frac{d f}{d s}(s) \mathbf{t}(s),
\end{aligned}
$$


with

$$
\begin{aligned}
g(s) & =-\widehat{\mathbf{R}}(s) \cdot \mathbf{t}(s), \\
\frac{d f}{d s}(s) & =\widehat{\mathbf{R}}(s) \cdot \mathbf{n}(s) .
\end{aligned}
$$

The functions $f(s), g(s)$, and $\mathbf{v}(s)$ are therefore completely specified by the boundary data; moreover, it can easily be checked that

$$
\widehat{\mathbf{R}}(s)=\mathbf{v}(s) \times \mathbf{e} ;
$$

thus the vectors $\mathbf{v}$ and $\widehat{\mathbf{R}}$ are mutually orthogonal and have the same magnitude.

The problem of finding a statically admissible stress field $\mathbf{T}$ reduces to that of finding a concave Airy function $\phi$ that satisfies the boundary conditions (8) and (9).

Notice that, for every $\left(\mathbf{x}_{1}, \mathbf{x}_{2}\right) \in \mathscr{H}$,

$$
\mathbf{R}\left(\mathbf{x}_{1}, \mathbf{x}_{2}\right)=\widehat{\mathbf{R}}\left(s_{2}\right)-\widehat{\mathbf{R}}\left(s_{1}\right)
$$

and

$$
\mathbf{M}\left(\mathbf{x}_{1}, \mathbf{x}_{2}\right)=\widehat{\mathbf{M}}\left(s_{2}\right)-\widehat{\mathbf{M}}\left(s_{1}\right)-\widehat{\mathbf{R}}\left(s_{1}\right) \times\left(\mathbf{x}_{2}-\mathbf{x}_{1}\right)
$$

where $\mathbf{x}_{i}=\mathbf{x}\left(s_{i}\right)$; using (11), (12), and (13) it can be immediately verified that (A1), $(\mathrm{A} 2)$, and $(\mathrm{A} 2)^{\prime}$, rewritten in terms of $f(s)$ and $\mathbf{v}(s)$ take the form:

$$
\begin{gathered}
\mathbf{v}\left(s_{1}\right)=\mathbf{v}\left(s_{2}\right), \\
f\left(s_{2}\right)-f\left(s_{1}\right)=\mathbf{v}\left(s_{1}\right) \cdot\left(\mathbf{x}_{2}-\mathbf{x}_{1}\right)=\mathbf{v}\left(s_{1}\right) \cdot \ell\left(\mathbf{x}_{1}, \mathbf{x}_{2}\right)\left|\mathbf{x}_{1}-\mathbf{x}_{2}\right| ; \\
{\left[f\left(s_{2}\right)-f\left(s_{1}\right)\right]\left|\mathbf{x}_{2}-\mathbf{x}_{1}\right|^{-1}<\mathbf{v}\left(s_{1}\right) \cdot \ell\left(\mathbf{x}_{1}, \mathbf{x}_{2}\right) ;} \\
{\left[\mathbf{v}\left(s_{2}\right)-\mathbf{v}\left(s_{1}\right)\right] \cdot \ell\left(\mathbf{x}_{1}, \mathbf{x}_{2}\right)<0 .}
\end{gathered}
$$

3. Proof of the main theorem. The proof is long and is more easily readable if presented by steps.

In Sec. 3.a we introduce, in a two-dimensional neighbourhood $\mathscr{S}_{\tau}$ of $\partial \Omega$, a local coordinate system built in such a way that one family of coordinate lines is straight and directed as the load $\mathbf{p}$. In Sec. 3.b we define a stress field $\mathbf{T}_{\tau}$ which is uniaxial in the direction of $\mathbf{p}$ and statically admissible in $\mathscr{S}_{\tau} \cap \Omega$ (Lemma 1). One of the main purposes of the subsequent analysis (Secs. 3.c and 3.d) is to show that the tractions

$$
\mathbf{p}_{\tau} \equiv-\mathbf{T}_{\tau} \cdot \mathbf{n}_{\tau} \quad \text { on } \partial\left(\Omega-\mathscr{S}_{\tau}\right) \equiv \partial \Omega_{\tau}
$$

are such that either (A1) or (A2) holds in $\mathscr{H}_{\tau} \equiv \partial \Omega_{\tau} \times \partial \Omega_{\tau}$. Here $\mathbf{n}_{\tau}$ is the outward (with respect to $\mathscr{S}_{\tau}$ ) unit normal to $\partial \Omega_{\tau}$.

In 3.c we show (Lemma 2) that, for a convenient choice of $t$, either (A1) or (A2) holds in an open neighbourhood $\mathscr{M}_{t}$ of the "principal diagonal" $\operatorname{diag} \mathscr{H}_{t}$ of $\mathscr{H}_{t}$,

$$
\operatorname{diag} \mathscr{H}_{t}=\left\{\left(\mathbf{y}_{1}, \mathbf{y}_{2}\right) \in \mathscr{H}_{t} \mid \mathbf{y}_{1}=\mathbf{y}_{2}\right\} .
$$

This neighbourhood has a counterimage $\mathscr{M}(t)$ in $\mathscr{H}$ obtained by "following" the family of coordinate lines that are directed as $\mathbf{p}$. 
In Sec. 3.d we investigate the function $d\left(\mathbf{x}_{1}, \mathbf{x}_{2}\right)$ (see formula (23)) which measures the distance of the central axis of $\mathbf{R}$ from $\mathbf{x}_{2}$ along the direction of $\mathbf{p}\left(\mathbf{x}_{2}\right)$. This function is defined over $\mathscr{H}_{2}$; we prove that

$$
\inf _{\mathscr{H}-\mathscr{M}(t)} d \equiv \bar{d}(t)>0 .
$$

In particular, this allows us to choose a value $\tau \in(0, \min \{t, \bar{d}(t)\})$ in such a way that the resultant $\mathbf{R}\left(\mathbf{y}_{1}, \mathbf{y}_{2}\right)$ is always compressive and its central axis intersects $\operatorname{seg}\left(\mathbf{y}_{1}, \mathbf{y}_{2}\right)$ or $\mathbf{R}\left(\mathbf{y}_{1}, \mathbf{y}_{2}\right)=0$. Thus either (A1) or (A2) holds on $\mathscr{H}_{\tau}-\mathscr{M}_{\tau}$. Recalling the result of Sec. 3.c and that $\mathscr{M}_{\tau} \subseteq \mathscr{M}_{t}$, we get that $\mathscr{H}_{\tau}=\mathscr{H}_{1 \tau} \cup \mathscr{H}_{2 \tau}$.

In Sec. 3.e, by using the results of 3.c and 3.d, we show that there exists a concave function $z=\psi_{\tau}^{+}(\mathbf{x})$ in $\Omega_{\tau}$ that takes the value $f_{\tau}$ over $\partial \Omega_{\tau}$; the boundary values $f_{\tau}, g_{\tau}$, and $\mathbf{v}_{\tau}$ are those determined, via (10) and (11), by $\mathbf{p}_{\tau}$ over $\partial \Omega_{\tau}$. This concave function has a continuous extension onto $\mathscr{S}_{\tau} \cap \Omega$ through the concave Airy function $z=\phi_{\tau}(\mathbf{x})$ corresponding to $\mathbf{T}_{\tau}$. The function

$$
z=\tilde{\phi}(\mathbf{x}) \equiv \begin{cases}\phi_{\tau}(\mathbf{x}), & \mathbf{x} \in \overline{\mathscr{S}}_{\tau} \cap \bar{\Omega}, \\ \psi_{\tau}^{+}(\mathbf{x}), & \mathbf{x} \in \Omega_{\tau}\end{cases}
$$

turns out to be concave all over $\Omega$ and to take the values $f$ and $\mathbf{v}$ on $\partial \Omega$; however, $z=\tilde{\phi}(\mathbf{x})$ is not necessarily smooth enough in $\bar{\Omega}_{\tau}$ to represent, via (7), a classical solution $\mathbf{T}$ to $(1)-(2)$.

In Sec. 3.f, by following an argument due to Giaquinta and Giusti [3], we prove that $z=\tilde{\phi}(\mathbf{x})$ can be regularized in order to provide a representation of the required solution.

3.a. The local coordinate system $\left(\xi_{1}, \xi_{2}\right)$. Since $\partial \Omega$ is compact and of class $C^{2}$ and $\mathbf{p} \in C^{2}(\partial \Omega)$, we can construct a coordinate patch in a neighbourhood of $\partial \Omega$, organized in such a way that one family of coordinate lines is straight and directed as the load $\mathbf{p}$ at the boundary (see Figure 2 on p. 738). We define local coordinates $\left(\xi_{1}, \xi_{2}\right)$ of $\mathbf{x} \in \mathbf{R}^{2}$ "close" to $\partial \Omega$ by

$$
\left\{\begin{array}{l}
\hat{\mathbf{x}}\left(\xi^{1}, \xi^{2}\right)=\mathbf{x}\left(\xi^{1}\right)+\xi^{2} \nu\left(\xi^{1}\right), \quad \xi^{2} \in[-\tau, \tau], \quad \xi^{1} \in[0, L), \\
\xi^{1}=s,
\end{array}\right.
$$

where

$$
\boldsymbol{\nu}(s)=|\mathbf{p}(\mathbf{x}(s))|^{-1} \mathbf{p}(\mathbf{x}(s)) .
$$

Since $\nu$ points inward in $\Omega$ (recall (ii) of the Main Theorem) we have

$$
\cos \alpha(s) \equiv \boldsymbol{\nu}(s) \cdot(-\mathbf{n}(s))>0, \quad \forall s \in[0, L) .
$$

In (16) $\tau$ is a positive constant chosen in such a way that the coordinate system never becomes singular. In this connection consider the limit

$$
\mathscr{R}(s) \equiv \lim _{t \rightarrow 0} \widetilde{\mathscr{R}}(s, t),
$$

where $\widetilde{R}(s, t)$ measures, up to the sign, the distance of $\mathbf{x}(s)$ from the point $C^{*}(s, t)$ of intersection of the two straight lines directed as $\nu(s)$ and $\nu(s+t)$ (see Fig. 2). If 


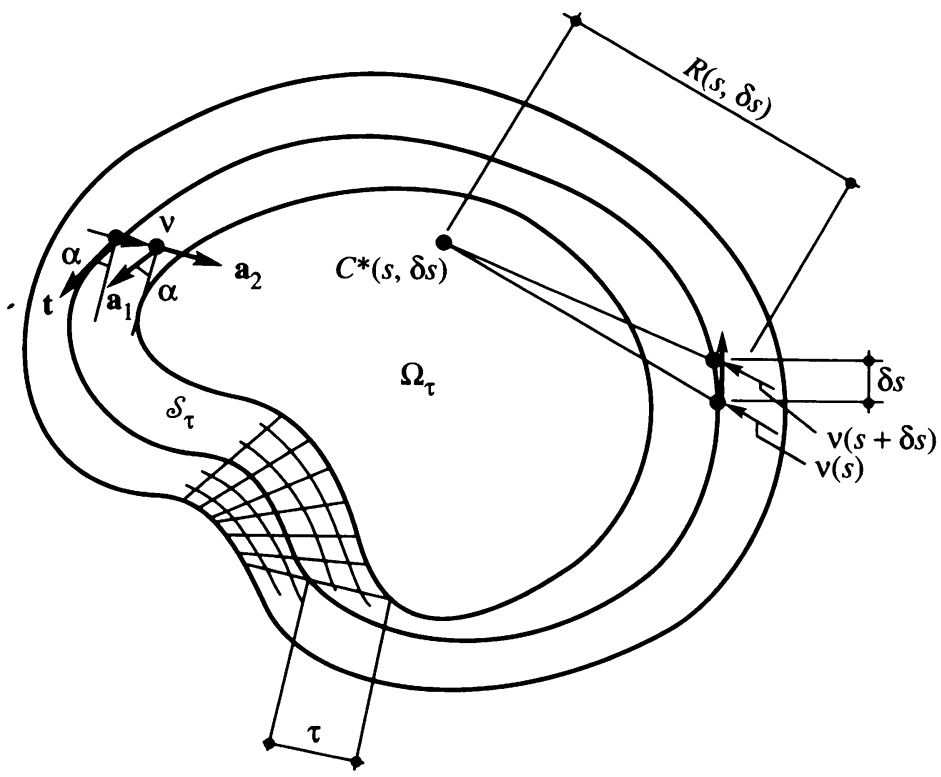

FIG. 2.

$\mathbf{p} \in C^{2}(\partial \Omega)$, then $\mathscr{R}(s) \in C^{1}([0, L))$ and never vanishes on $[0, L)$. The function $\mathscr{R}(s)$ is related to the "bending" of the field $\boldsymbol{\nu}(s)$ along $\partial \Omega$ by the equation

$$
\frac{d \nu}{d s}=\frac{\cos \alpha(s)}{\mathscr{R}(s)} \sigma(s),
$$

where $\boldsymbol{\sigma} \equiv \mathbf{e} \times \boldsymbol{\nu}$ (see Appendix I).

Clearly $\min _{[0, L)}|\mathscr{R}(s)| \equiv \overline{\mathscr{R}}>0$. From (34) and (36) in the Appendix, we obtain the following necessary condition for the coordinate system $\left(\xi^{1}, \xi^{2}\right)$ to be well defined:

$$
0<\tau<\overline{\mathscr{R}} \text {. }
$$

In particular, this choice of $\tau$ implies the positiveness of the ratio $\left(\mathscr{R}\left(\xi^{1}\right)-\xi^{2}\right) / \mathscr{R}\left(\xi^{1}\right)$.

For any $\tau$ satisfying (19) we define a neighbourhood $\mathscr{S}_{\tau}$ of $\partial \Omega$ as follows:

$$
\mathscr{S}_{\tau} \equiv\left\{\mathbf{x}=\hat{\mathbf{x}}\left(\xi^{1}, \xi^{2}\right)\left|0 \leq \xi^{1}<L,\right| \xi^{2} \mid<\tau\right\} .
$$

Since $\partial \Omega$ is compact and of class $C^{2}$, it satisfies a uniform sphere condition; that is, at each point $\mathbf{y} \in \partial \Omega$ there exist two open balls $B_{1}$ and $B_{2}$ such that $\bar{B}_{1} \cap\left(\mathbf{R}^{2}-\Omega\right)=$ $\{\mathbf{y}\}, \bar{B}_{2} \cap \bar{\Omega}=\{\mathbf{y}\}$ and the radii of the balls are uniformly bounded from below by a positive constant $\mu$. Evidently $\mu^{-1}$ bounds the curvature of $\partial \Omega$.

Then, if $\tau$ is less than $\mu$, the curves $\mathbf{x}=\hat{\mathbf{x}}\left(\xi^{1}, \pm \tau\right)$ are simple, of class $C^{2}$, and represent the boundary of $\mathscr{S}_{\tau}$. Therefore,

$$
\tau<\min \{\overline{\mathscr{R}}, \mu\}
$$

is a necessary and sufficient condition for the coordinate system $\left(\xi^{1}, \xi^{2}\right)$ to be well defined. 
Let us finally set $\Omega_{\tau} \equiv \Omega-\mathscr{S}_{\tau}, \mathbf{n}_{\tau}$ the outward (with respect to $\mathscr{S}_{\tau}$ ) unit normal along $\partial \Omega_{\tau}$, and $\mathscr{H}_{\tau} \equiv \partial \Omega_{\tau} \times \partial \Omega_{\tau}$.

3.b. A statically admissible stress field in $\mathscr{S}_{\tau} \cap \Omega$. Let us define over $\mathscr{S}_{\tau}$ the stress field

$$
\mathbf{T}_{\tau}\left(\xi^{1}, \xi^{2}\right) \equiv-\mathscr{R}\left(\xi^{1}\right) \frac{\left|\mathbf{p}\left(\mathbf{x}\left(\xi^{1}\right)\right)\right|}{\left(\mathscr{R}\left(\xi^{1}\right)-\xi^{2}\right) \cos \alpha\left(\xi^{1}\right)} \boldsymbol{\nu}\left(\xi^{1}\right) \otimes \boldsymbol{\nu}\left(\xi^{1}\right) .
$$

This stress field is compressive, uniaxial, verifies condition $(1)_{3}$ over $\partial \Omega$, and defines $\mathbf{p}_{\tau}$ via (15).

Lemma 1. The restriction of $\mathbf{T}_{\tau}$ onto $\mathscr{S}_{\tau} \cap \Omega$ is equilibrated with the load $\mathbf{p}$.

The proof of this Lemma is presented in Appendix I.

As a consequence of Lemma $1 \mathbf{T}_{\tau}$ is statically admissible in $\mathscr{S}_{\tau} \cap \Omega$.

3.c. The open neighbourhood $\mathscr{M}_{t}$ of $\operatorname{diag} \mathscr{H}_{t}$.

Lemma 2. If the hypotheses of the Main Theorem hold, then there exists a number $t>0$ and an open neighbourhood $\mathscr{M}_{t}$ of $\operatorname{diag} \mathscr{H}_{t}$ such that

$$
\mathscr{M}_{t} \subset \mathscr{H}_{1 t} \cup \mathscr{H}_{2 t},
$$

where $\mathscr{H}_{i t}$ denotes the subset of $\mathscr{H}_{t}$ that verifies $(\mathrm{A} i)$, with $i=1,2$.

Proof. Let us consider the following neighbourhood of $\partial \Omega$ :

$$
\Sigma_{\theta}=\left\{\mathbf{x} \in \mathbf{R}^{2} \mid \operatorname{dist}(\mathbf{x}, \partial \Omega)<\theta\right\},
$$

where $\theta$ is a positive number. Since $\mathscr{S}_{\tau_{1}}$ with $\tau_{1}<\min \{\overline{\mathscr{R}}, \mu\}$ is an open neighbourhood of $\partial \Omega$, there exists a number $t>0$ such that

$$
\Sigma_{3 t} \subseteq \mathscr{S}_{\tau_{1}} \text {. }
$$

In the sequel of the proof $t$ is thought of as an arbitrarily fixed number for which (22) is satisfied. Consider now $\mathbf{y} \in \mathscr{S}_{t}$; then, by definition (16),

$$
\mathbf{y}=\mathbf{x}+\alpha \boldsymbol{\nu},
$$

with $\mathbf{x} \in \partial \Omega$ and $|\alpha|<t$. Then we have

$$
\operatorname{dist}(\mathbf{y}, \partial \Omega) \leq t .
$$

Let us also consider the following subset of $\mathscr{H}$ :

$$
\mathscr{M}(t)=\left\{\left(\mathbf{x}_{1}, \mathbf{x}_{2}\right) \in \mathscr{H}|| \mathbf{x}_{1}-\mathbf{x}_{2} \mid<t\right\} ;
$$

to this set corresponds, via (16), the set

$$
\mathscr{M}_{t}=\left\{\left(\mathbf{y}_{1}, \mathbf{y}_{2}\right) \in \mathscr{H}_{t} \mid \mathbf{y}_{i}=\mathbf{x}_{i}+t \nu_{i}, i=1,2 \text { and }\left(\mathbf{x}_{1}, \mathbf{x}_{2}\right) \in \mathscr{M}(t)\right\},
$$

where $\boldsymbol{\nu}_{i} \equiv \boldsymbol{\nu}\left(s_{i}\right)$.

The open ball $\mathscr{C}_{\rho}(\overline{\mathbf{z}})$ with center in $\overline{\mathbf{z}}=\frac{1}{2}\left(\mathbf{y}_{1}+\mathbf{y}_{2}\right)$, where $\left(\mathbf{y}_{1}, \mathbf{y}_{2}\right) \in \mathscr{M}_{t}$, and radius $\rho=\frac{1}{2}\left|\mathbf{y}_{1}-\mathbf{y}_{2}\right|$ is contained in $\Sigma_{3 t}$ and therefore in $\mathscr{S}_{\tau_{1}}$. Indeed, for every $\mathbf{z} \in \mathscr{C}_{\rho}(\overline{\mathbf{z}})$ we have

$$
\left|\mathbf{z}-\mathbf{x}_{1}\right| \leq|\mathbf{z}-\overline{\mathbf{z}}|+\left|\overline{\mathbf{z}}-\mathbf{x}_{1}\right|
$$


and since

$$
\begin{gathered}
|\mathbf{z}-\overline{\mathbf{z}}| \leq\left|\mathbf{y}_{2}-\mathbf{y}_{1}\right| / 2 \leq 3 t / 2 \\
\left|\overline{\mathbf{z}}-\mathbf{x}_{1}\right| \leq\left|\left(\mathbf{y}_{2}-\mathbf{y}_{1}\right) / 2-\mathbf{x}_{1}\right| \leq\left(\left|\mathbf{y}_{2}-\mathbf{x}_{2}\right|+\left|\mathbf{x}_{2}-\mathbf{x}_{1}\right|+\left|\mathbf{y}_{1}-\mathbf{x}_{1}\right|\right) / 2<3 t / 2,
\end{gathered}
$$

then

$$
\operatorname{dist}(\mathbf{z}, \partial \Omega) \leq\left|\mathbf{z}-\mathbf{x}_{1}\right|<\frac{3 t}{2}+\frac{3 t}{2}=3 t .
$$

In view of (22), we have proved the inclusion $\mathscr{C}_{\rho}(\overline{\mathbf{z}}) \subset \mathscr{S}_{\tau}$. Consider the restriction $\overline{\mathbf{T}}_{\tau}$ of $\mathbf{T}_{\tau}$ to $\mathscr{C}_{\rho}(\overline{\mathbf{z}})$; since $\overline{\mathbf{T}}_{\tau}$ is a s.a.f. then, from Proposition 1, either (A1) or (A2) holds for every $\left(\mathbf{z}_{1}, \mathbf{z}_{2}\right) \in \partial \mathscr{C}_{\rho}(\overline{\mathbf{z}}) \times \partial \mathscr{C}_{\rho}(\overline{\mathbf{z}})$ and hence for $\left(\mathbf{y}_{1}, \mathbf{y}_{2}\right) \in \mathscr{M}_{t}$.

3.d. The function $d\left(\mathbf{x}_{1}, \mathbf{x}_{2}\right)$ over $\mathscr{H}_{2}-\mathscr{M}(t)$. Let us denote by

$$
d\left(\mathbf{x}_{1}, \mathbf{x}_{2}\right)=\frac{-\mathbf{M}\left(\mathbf{x}_{1}, \mathbf{x}_{2}\right) \cdot \mathbf{e}}{\left|\mathbf{R}\left(\mathbf{x}_{1}, \mathbf{x}_{2}\right) \cdot \tilde{\boldsymbol{\sigma}}\left(\mathbf{x}_{2}\right)\right|}
$$

where $\tilde{\boldsymbol{\sigma}}$ is defined through the relation $\boldsymbol{\sigma}(s)=\tilde{\boldsymbol{\sigma}} \circ \mathbf{x}(s)$. We want to investigate the behavior of $d\left(\mathbf{x}_{1}, \mathbf{x}_{2}\right)$ over $\overline{\mathscr{H}}_{2}-\mathscr{M}(t)$. First of all notice that the hypothesis (ii) of the Main Theorem implies

$$
\begin{gathered}
\stackrel{\circ}{\mathscr{H}}_{1}=\varnothing, \\
\forall\left(\mathbf{x}_{1}, \mathbf{x}_{2}\right) \in \mathscr{H}_{1}, \exists \mathscr{I}\left(\mathbf{x}_{2}\right) \cap \partial \Omega:\left(\mathbf{x}_{1}, \mathbf{x}\right) \notin \mathscr{H}_{1} \forall x \in \mathscr{I}\left(\mathbf{x}_{2}\right)-\left\{\mathbf{x}_{2}\right\} .
\end{gathered}
$$

Indeed, for $\left(\overline{\mathbf{x}}_{1}, \overline{\mathbf{x}}_{2}\right) \in \stackrel{\circ}{\mathscr{H}}{ }_{1}\left(\mathbf{x}_{i} \equiv \mathbf{x}\left(s_{i}\right)\right)$ let $\mathscr{U} \subseteq \stackrel{\circ}{\mathscr{H}}$, be an open neighbourhood of $\left(\overline{\mathbf{x}}_{1}, \overline{\mathbf{x}}_{2}\right)$. For every pair $\left(\mathbf{x}_{1}, \mathbf{x}_{2}\right) \in \mathscr{U}$ we have

$$
\mathbf{R}\left(\mathbf{x}_{1}, \mathbf{x}_{2}\right)=\mathbf{M}\left(\mathbf{x}_{1}, \mathbf{x}_{2}\right)=0 \text {. }
$$

Because of definition (3) we have

$$
\mathbf{R}\left(\mathbf{x}\left(\bar{s}_{2}-h\right), \mathbf{x}\left(\bar{s}_{2}+h\right)\right)=\mathbf{R}\left(\mathbf{x}_{1}, \mathbf{x}\left(\bar{s}_{2}+h\right)\right)-\mathbf{R}\left(\mathbf{x}_{1}, \mathbf{x}\left(\bar{s}_{2}-h\right)\right) ;
$$

since both terms on the right-hand side vanish for every $h$, we would get

$$
\mathbf{p}\left(\overline{\mathbf{x}}_{2}\right)=\lim _{h \rightarrow 0} \frac{\mathbf{R}\left(\mathbf{x}\left(\bar{s}_{2}-h\right), \mathbf{x}\left(\bar{s}_{2}+h\right)\right)}{2 h}=0,
$$

which is a contradiction with $\Gamma_{0}=\varnothing$. Consequently $\mathscr{H}_{1}=\partial \mathscr{H}_{1}$ and, if $\mathscr{H}=\mathscr{H}_{1} \cup \mathscr{H}_{2}$, $\partial \mathscr{H}_{2}=\mathscr{H}_{1}$.

We get exactly the same contradiction if we assume the existence of an open neighbourhood $\mathscr{I}\left(\overline{\mathbf{x}}_{2}\right) \cap \partial \Omega$ of $\overline{\mathbf{x}}_{2}$ such that

$$
\mathbf{R}\left(\overline{\mathbf{x}}_{1}, \mathbf{x}_{2}\right)=\mathbf{M}\left(\overline{\mathbf{x}}_{1}, \mathbf{x}_{2}\right)=0
$$

for every $\mathbf{x}_{2} \in \mathscr{I}\left(\overline{\mathbf{x}}_{2}\right) \cap \partial \Omega$.

Clearly $d$ turns out to be an indeterminate form of type $\frac{0}{0}$ if $\left(\mathbf{x}_{1}, \mathbf{x}_{2}\right) \in \partial \mathscr{H}_{2}$. We want to show that $d$ can be extended, in the sense specified by Lemma 4 , to all of $\overline{\mathscr{H}}_{2}-\mathscr{M}(t)$, remaining strictly positive.

It should be noticed that $\mathbf{R} \cdot \tilde{\boldsymbol{\sigma}}$ may be zero when $\left(\mathbf{x}_{1}, \mathbf{x}_{2}\right) \in \stackrel{\circ}{\mathscr{H}}{ }_{2}$. However, in this case $\mathbf{M}\left(\mathbf{x}_{1}, \mathbf{x}_{2}\right) \cdot \mathbf{e}<0$ and therefore $d$ may become unbounded in $\stackrel{\mathscr{H}}{2}_{2}$. This circumstance does not matter within our discussion since we are interested only in the minimum value $\bar{d}$ of $d$ in $\overline{\mathscr{H}}_{2}-\mathscr{M}(t)$, which is actually positive (Lemma 4$)$. As a preliminary step to this purpose we need to prove the following. 
Lemma 3. Let $\left(\mathbf{x}_{1}, \mathbf{x}_{2}\right) \in\left(\mathscr{H}_{1}-\operatorname{diag} \mathscr{H}\right)$. If the hypotheses of the main theorem hold, then we have

$$
\boldsymbol{\nu}\left(s_{2}\right) \times\left(\mathbf{x}_{2}-\mathbf{x}_{1}\right)=0 .
$$

Proof. As we have seen before $\partial \mathscr{H}_{2}=\mathscr{H}_{1}$ if $\mathscr{H}=\mathscr{H}_{1} \cup \mathscr{H}_{2}$. Given $\left(\mathbf{x}_{1}, \mathbf{x}_{2}\right) \in \partial \mathscr{H}_{2}$, because of (25), we can find two points $\overline{\mathbf{x}}^{\prime}, \overline{\mathbf{x}}^{\prime \prime}$ such that $\left(\mathbf{x}_{1}, \overline{\mathbf{x}}^{\prime}\right),\left(\mathbf{x}_{1}, \overline{\mathbf{x}}^{\prime \prime}\right) \in \mathscr{H}_{2}$ and

$$
\begin{aligned}
-\mathbf{R}\left(\mathbf{x}_{1}, \mathbf{x}^{\prime \prime}\right) \cdot \mathbf{r}\left(\mathbf{x}_{1}, \mathbf{x}^{\prime \prime}\right)<0 & \forall \mathbf{x}^{\prime \prime} \in \gamma\left(\mathbf{x}_{2}, \overrightarrow{\mathbf{x}}^{\prime \prime}\right), \\
-\mathbf{R}\left(\mathbf{x}_{1}, \mathbf{x}^{\prime}\right) \cdot \mathbf{r}\left(\mathbf{x}_{1}, \mathbf{x}^{\prime}\right)<0 & \forall \mathbf{x}^{\prime} \in \gamma\left(\overrightarrow{\mathbf{x}}^{\prime}, \mathbf{x}_{2}\right) .
\end{aligned}
$$

By definition (3)

$$
\begin{aligned}
\mathbf{R}\left(\mathbf{x}_{1}, \mathbf{x}^{\prime \prime}\right) & =\mathbf{R}\left(\mathbf{x}_{2}, \mathbf{x}^{\prime \prime}\right), \\
\mathbf{R}\left(\mathbf{x}_{1}, \mathbf{x}^{\prime}\right) & =\mathbf{R}\left(\mathbf{x}_{2}, \mathbf{x}^{\prime}\right) .
\end{aligned}
$$

Therefore, we can also write

$$
\begin{aligned}
-\mathbf{R}\left(\mathbf{x}_{2}, \mathbf{x}^{\prime \prime}\right) \cdot \mathbf{r}\left(\mathbf{x}_{1}, \mathbf{x}^{\prime \prime}\right)<0 & \forall \mathbf{x}^{\prime \prime} \in \gamma\left(\mathbf{x}_{2}, \overrightarrow{\mathbf{x}}^{\prime \prime}\right), \\
-\mathbf{R}\left(\mathbf{x}_{2}, \mathbf{x}^{\prime}\right) \cdot \mathbf{r}\left(\mathbf{x}_{1}, \mathbf{x}^{\prime}\right)<0 & \forall \mathbf{x}^{\prime} \in \gamma\left(\overrightarrow{\mathbf{x}}^{\prime}, \mathbf{x}_{2}\right) .
\end{aligned}
$$

Dividing the last inequalities by $\left|\mathbf{x}^{\prime \prime}-\mathbf{x}_{2}\right|$ and $\left|\mathbf{x}^{\prime}-\mathbf{x}_{2}\right|$, respectively, and passing to the limit for $\mathbf{x}^{\prime}, \mathbf{x}^{\prime \prime} \rightarrow \mathbf{x}_{2}$, we get at the same time

$$
\begin{aligned}
\mathbf{p}\left(\mathbf{x}_{2}\right) \cdot \mathbf{r}\left(\mathbf{x}_{1}, \mathbf{x}_{2}\right) & \leq 0, \\
-\mathbf{p}\left(\mathbf{x}_{2}\right) \cdot \mathbf{r}\left(\mathbf{x}_{1}, \mathbf{x}_{2}\right) & \leq 0 .
\end{aligned}
$$

Since $\mathbf{p}\left(\mathbf{x}_{2}\right) \neq 0$ we obtain $\boldsymbol{\nu}\left(s_{2}\right) \cdot \mathbf{r}\left(\mathbf{x}_{1}, \mathbf{x}_{2}\right)=0$, which is equivalent to (24).

Let us put

$$
\begin{gathered}
D\left(s_{1}, s_{2}\right) \equiv d\left(\mathbf{x}\left(s_{1}\right), \mathbf{x}\left(s_{2}\right)\right), \quad \mathscr{D} \equiv[0, L) \times[0, L), \\
\mathscr{D}_{i} \equiv\left\{\left(s_{1}, s_{2}\right) \in \mathscr{D} \mid\left(\mathbf{x}_{1}, \mathbf{x}_{2}\right) \in \mathscr{H}_{i}\right\} .
\end{gathered}
$$

Because of (i) of the Main Theorem and of the injectivity of $\mathbf{x}=\mathbf{x}(s)$ onto $\partial \Omega$ we have that

$$
\mathscr{D}=\mathscr{D}_{1} \cup \mathscr{D}_{2} .
$$

We think of $\mathscr{D}$ as endowed with the topology induced from that of $\mathscr{H}$ via $\mathbf{x}=\mathbf{x}(s)$. Recalling what we said above about the function $d$, it is clear that the function $D$ is defined in $\mathscr{D}_{2}$. Moreover, since $\stackrel{\mathscr{H}}{1}_{1}=\varnothing$ and $\partial \mathscr{H}_{2}=\partial \mathscr{H}_{1}=\mathscr{H}_{1}$, we also have $\mathscr{\mathscr { D }}_{1}=\varnothing$ and $\partial \mathscr{D}_{2}=\partial \mathscr{D}_{1}=\mathscr{D}_{1}$. Finally, let $\mathscr{E}(t)$ denote the counterimage of $\mathscr{M}(t)$ via $\mathbf{x}=\mathbf{x}(s)$.

LEMma 4. If the hypotheses of the Main Theorem hold, then for every point $\left(s_{1}^{0}, s_{2}^{0}\right)$ $\in\left(\mathscr{D}_{1}-\operatorname{diag} \mathscr{D}\right)$

$$
D_{m}\left(s_{1}^{0}, s_{2}^{0}\right) \equiv \min _{\left(s_{1}, s_{2}\right) \rightarrow\left(s_{1}^{0}, s_{2}^{0}\right)} D\left(s_{1}, s_{2}\right)>0 .
$$

In particular, $\bar{d}(t) \equiv \inf _{\mathscr{Z}-\mathscr{E}(t)}(D)>0$. 
Proof. To simplify notation we write

$$
\begin{aligned}
\mathbf{S} & \equiv\left(s_{1}, s_{2}\right), \\
\mathbf{W} & \equiv\left(w_{1}, w_{2}\right)=\mathbf{S}-\mathbf{S}_{0}, \\
H(\mathbf{S}) & \equiv-\mathbf{M}\left[\mathbf{x}\left(s_{1}\right), \mathbf{x}\left(s_{2}\right)\right] \cdot \mathbf{e}, \\
K(\mathbf{S}) & \equiv \mathbf{R}\left[\mathbf{x}\left(s_{1}\right), \mathbf{x}\left(s_{2}\right)\right] \cdot \boldsymbol{\sigma}\left(s_{2}\right), \\
\check{\mathbf{r}}\left(s_{1}, s_{2}\right) & \equiv \mathbf{r}\left[\mathbf{x}\left(s_{1}\right), \mathbf{x}\left(s_{2}\right)\right], \\
\check{\ell}\left(s_{1}, s_{2}\right) & \equiv \ell\left[\mathbf{x}\left(s_{1}\right), \mathbf{x}\left(s_{2}\right)\right] .
\end{aligned}
$$

If $\mathbf{S}_{0} \in\left(\mathscr{D}_{1}-\operatorname{diag} \mathscr{D}\right), D\left(\mathbf{S}_{0}\right)$ turns out to be an indeterminate form $\frac{0}{0}$. However, we can apply Taylor's formula to both the numerator $H(\mathbf{S})$ and the denominator $K(\mathbf{S})$ of $d$ using just $\mathbf{S}_{0}$ as an initial point.

To justify the use of Taylor's formula to the functions $H$ and $K$ we observe that since $\mathbf{p}$ and $\partial \Omega$ are of class $C^{2}$, both $\mathbf{R}\left[\mathbf{x}\left(s_{1}\right), \mathbf{x}\left(s_{2}\right)\right]$ and $\mathbf{M}\left[\mathbf{x}\left(s_{1}\right), \mathbf{x}\left(s_{2}\right)\right]$ are of class $C^{2}$ on $\mathscr{D}$; this can easily be checked by using (3) and (4). Therefore, $H(\mathbf{S})$ is of class $C^{2}$; as far as $K(\mathbf{S})$ is concerned, we shall prove (formula (31) in the Appendix) that $d \boldsymbol{\nu} / d s=\lambda \boldsymbol{\sigma}$ where $\lambda(s)=(\cos \alpha(s)) / \mathscr{R}(s)$; recalling that $\boldsymbol{\sigma}=\mathbf{e} \times \boldsymbol{\nu}$ we easily obtain that

$$
\frac{d \sigma}{d s}=-\lambda \nu
$$

Therefore, recalling our hypotheses on the regularity of $\partial \Omega$ and $\mathbf{p}$, the function $\lambda$ is of class $C^{1}$ and, consequently, $\sigma$ is of class $C^{2}$. This allows us to conclude that $K(\mathbf{S})$ is also of class $C^{2}$.

The Taylor formula for $H(\mathbf{S})$ up to the second order is

$$
H(\mathbf{S})=H\left(\mathbf{S}_{0}\right)+\mathbf{W} \cdot \nabla H\left(\mathbf{S}_{0}\right)+\frac{1}{2} \sum_{i, j} \partial_{i j} H\left(\mathbf{S}_{0}\right) w_{i} w_{j}+o\left(|\mathbf{W}|^{2}\right)
$$

and similarly for $K(\mathbf{S})$.

By recalling formula (4) we get

$$
\begin{aligned}
& \partial_{1} H(\mathbf{S})=\mathbf{p}\left[\mathbf{x}\left(s_{1}\right)\right] \times\left[\mathbf{x}\left(s_{2}\right)-\mathbf{x}\left(s_{1}\right)\right] \cdot \mathbf{e}, \\
& \partial_{2} H(\mathbf{S})=\left[\widehat{\mathbf{R}}\left(s_{1}\right)-\widehat{\mathbf{R}}\left(s_{2}\right)\right] \times \mathbf{t}\left(s_{2}\right) \cdot \mathbf{e} .
\end{aligned}
$$

Because of Lemma 3, hypothesis (A1) and formula (13), we have

$$
\boldsymbol{\nabla} H\left(\mathbf{S}_{0}\right)=0 .
$$

Similarly we get

$$
\begin{aligned}
& \partial_{1} K(\mathbf{S})=-\mathbf{p}\left(\mathbf{x}\left(s_{1}\right)\right) \cdot\left[\mathbf{e} \times \boldsymbol{\nu}\left(s_{2}\right)\right], \\
& \partial_{2} K(\mathbf{S})=\left[\widehat{\mathbf{R}}\left(s_{2}\right)-\widehat{\mathbf{R}}\left(s_{1}\right)\right] \cdot\left(\mathbf{e} \times \frac{d}{d s_{2}} \boldsymbol{\nu}\left(s_{2}\right)\right) .
\end{aligned}
$$

Thus, as before,

$$
\boldsymbol{\nabla} K\left(\mathbf{S}_{0}\right)=0 .
$$


From (23) we have

$$
D(\mathbf{S})=\frac{\sum \partial_{i j} H\left(\mathbf{S}_{0}\right) w_{i} w_{j}+o\left(|\mathbf{W}|^{2}\right)}{\left|\sum \partial_{i j} K\left(\mathbf{S}_{0}\right) w_{i} w_{j}+o\left(|\mathbf{W}|^{2}\right)\right|} .
$$

Since $D$ is strictly positive and smooth in $\mathscr{D}_{2}$ we have

$$
\min _{\mathbf{S} \rightarrow \mathbf{S}_{0}} \lim D(\mathbf{S}) \geq 0 \text {. }
$$

Let $\left(\mathbf{S}_{n}\right)$ be a sequence converging to $\mathbf{S}_{0}$ and such that

$$
\lim _{n \rightarrow \infty} D\left(\mathbf{S}_{n}\right)=\min _{\mathbf{S} \rightarrow \mathbf{S}_{0}} D(\mathbf{S}) \equiv D_{m}\left(\mathbf{S}_{0}\right) \text {. }
$$

We want to show that $D_{m}\left(\mathbf{S}_{0}\right)>0$. First of all notice that

$$
\lim _{n \rightarrow \infty} D\left(\mathbf{S}_{n}\right)=\lim _{n \rightarrow \infty} \frac{\sum \partial_{i j} H\left(\mathbf{S}_{0}\right) w_{n i} w_{n j}}{\left|\sum \partial_{i j} K\left(\mathbf{S}_{0}\right) w_{n i} w_{n j}\right|} .
$$

Moreover, the two matrices with entries $\partial_{i j} H\left(\mathbf{S}_{0}\right)$ and $\left|\partial_{i j} K\left(\mathbf{S}_{0}\right)\right|$, respectively, are both symmetric; recalling the hypothesis (iii) of the Main Theorem, we have

$$
\sum \partial_{i j} H\left(\mathbf{S}_{0}\right) w_{n i} w_{n j} \geq \beta\left(\mathbf{S}_{0}\right)\left|\mathbf{W}_{n}\right|^{2}>0 \text {. }
$$

Moreover,

$$
\left|\sum \partial_{i j} K\left(\mathbf{S}_{0}\right) w_{n i} w_{n j}\right| \leq \delta\left(\mathbf{S}_{0}\right)\left|\mathbf{W}_{n}\right|^{2}
$$

where $\delta\left(\mathbf{S}_{0}\right) \equiv \max \left\{2\left|\partial_{i j} K\left(\mathbf{S}_{0}\right)\right| \mid i, j=1,2\right\}<\infty$. Therefore,

$$
D_{m}\left(\mathbf{S}_{0}\right) \geq \frac{\beta\left(\mathbf{S}_{0}\right)}{\delta\left(\mathbf{S}_{0}\right)}>0, \quad \forall \mathbf{S}_{0} \in \mathscr{D}_{1}-\operatorname{diag} \mathscr{D} .
$$

Consequently $D_{m}\left(\mathbf{S}_{0}\right)$ is strictly positive on all of $\overline{\mathscr{D}}_{2}-\mathscr{E}(t)$. Since $\mathscr{M}(t)$ is open, also $\mathscr{E}(t)$ is open, and therefore $\overline{\mathscr{D}}_{2}-\mathscr{E}(t)$ is a compact set. This implies that

$$
\inf _{\overline{\mathscr{D}}_{2}-\mathscr{E}(t)} D(\mathbf{S})=\inf _{\mathscr{H}_{2}-\mathscr{M}(t)} d(\mathbf{S}) \equiv \bar{d}(t)>0
$$

and Lemma 4 is thus completely proved.

We are now ready to show that a value $\tau=\tau_{2}$ can always be chosen in such a way that either (A1) or (A2) is satisfied by $\left(\mathbf{y}_{1}, \mathbf{y}_{2}\right) \in \mathscr{H}_{\tau}$, that is, $\mathscr{H}_{\tau}=\mathscr{H}_{1 \tau} \cup \mathscr{H}_{2 \tau}$. If we choose

$$
\tau=\tau_{2} \leq \min \{t, \bar{d}(t)\},
$$

either $\left(\mathbf{y}_{1}, \mathbf{y}_{2}\right) \in \mathscr{M}_{\tau} \subseteq \mathscr{M}_{t}$-and in that case Lemma 2 applies-or $\left(\mathbf{y}_{1}, \mathbf{y}_{2}\right) \in \mathscr{H}_{\tau}-$ $\mathscr{M}_{\tau}$. If the latter case occurs, notice first that since $\mathbf{T}_{\tau}$ is uniaxial in the direction of the load, no tractions are transmitted across the segments $\mathbf{x}(s)+\lambda(\mathbf{y}(s)-\mathbf{x}(s))$, $\lambda \in[0,1]$, that is (see the Appendix) $\mathbf{T}_{\lambda} \cdot \boldsymbol{\sigma}=0$. Therefore,

$$
\mathbf{R}\left(\mathbf{x}_{1}, \mathbf{x}_{2}\right)=\mathbf{R}\left(\mathbf{y}_{1}, \mathbf{y}_{2}\right) \text { and } \mathbf{M}\left(\mathbf{x}_{1}, \mathbf{x}_{2}\right)=\mathbf{M}\left(\mathbf{y}_{1}, \mathbf{y}_{2}\right)+\mathbf{R}\left(\mathbf{y}_{1}, \mathbf{y}_{2}\right) \times\left(\mathbf{x}_{2}-\mathbf{y}_{2}\right),
$$

for every $\left(\mathbf{x}_{1}, \mathbf{x}_{2}\right) \in \mathscr{H}$, where $\mathbf{x}_{i}=\mathbf{x}\left(s_{i}\right)$ and $\mathbf{y}_{i}=\mathbf{y}\left(s_{i}\right)=\mathbf{x}\left(s_{i}\right)+\tau \nu\left(s_{i}\right)$. Thus

$$
\left(\mathbf{x}_{1}, \mathbf{x}_{2}\right) \in \mathscr{H}_{1} \Leftrightarrow\left(\mathbf{y}_{1}, \mathbf{y}_{2}\right) \in \mathscr{H}_{1 \tau} \text {. }
$$




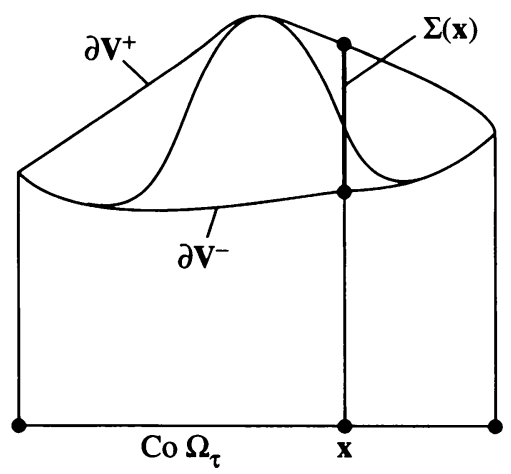

FIG. 3.

Moreover, if $\left(\mathbf{x}_{1}, \mathbf{x}_{2}\right) \in \mathscr{H}_{2}$ we have

$$
\bar{d}(t) \leq \frac{-\left(\mathbf{M}\left(\mathbf{y}_{1}, \mathbf{y}_{2}\right) \cdot \mathbf{e}+\mathbf{R}\left(\mathbf{y}_{1}, \mathbf{y}_{2}\right) \times\left(\mathbf{x}_{2}-\mathbf{y}_{2}\right) \cdot \mathbf{e}\right)}{\left|\mathbf{R}\left(\mathbf{y}_{1}, \mathbf{y}_{2}\right) \cdot \tilde{\boldsymbol{\sigma}}\left(\mathbf{y}_{2}\right)\right|},
$$

since $\tilde{\boldsymbol{\sigma}}\left(\mathbf{x}_{2}\right)=\tilde{\boldsymbol{\sigma}}\left(\mathbf{y}_{2}\right)$. Thus, were

$$
\bar{d}(t)+\frac{\mathbf{R}\left(\mathbf{y}_{1}, \mathbf{y}_{2}\right) \times\left(\mathbf{x}_{2}-\mathbf{y}_{2}\right) \cdot \mathbf{e}}{\left|\mathbf{R}\left(\mathbf{y}_{1}, \mathbf{y}_{2}\right) \cdot \tilde{\boldsymbol{\sigma}}\left(\mathbf{y}_{2}\right)\right|}>0,
$$

we would get $\left(\mathbf{y}_{1}, \mathbf{y}_{2}\right) \in \mathscr{H}_{2 \tau}$. To prove this notice that since

$$
\frac{\mathbf{R}\left(\mathbf{y}_{1}, \mathbf{y}_{2}\right) \times\left(\mathbf{x}_{2}-\mathbf{y}_{2}\right) \cdot \mathbf{e}}{\left|\mathbf{R}\left(\mathbf{y}_{1}, \mathbf{y}_{2}\right) \cdot \tilde{\boldsymbol{\sigma}}\left(\mathbf{y}_{2}\right)\right|}=-\tau \frac{\mathbf{R}\left(\mathbf{y}_{1}, \mathbf{y}_{2}\right) \cdot \tilde{\boldsymbol{\sigma}}\left(\mathbf{y}_{2}\right)}{\left|\mathbf{R}\left(\mathbf{y}_{1}, \mathbf{y}_{2}\right) \cdot \tilde{\boldsymbol{\sigma}}\left(\mathbf{y}_{2}\right)\right|},
$$

then

$$
\bar{d}(t) \pm \tau \leq \frac{-\mathbf{M}\left(\mathbf{y}_{1}, \mathbf{y}_{2}\right) \cdot \mathbf{e}}{\left|\mathbf{R}\left(\mathbf{y}_{1}, \mathbf{y}_{2}\right) \cdot \tilde{\boldsymbol{\sigma}}\left(\mathbf{y}_{2}\right)\right|},
$$

and because $\tau<\bar{d}(t)$, the right-hand side of the last inequality is always positive also when $\mathbf{R} \cdot \boldsymbol{\sigma}>0$.

3.e. Construction of a concave function $z=\tilde{\phi}(\mathbf{x})$ over $\Omega$. Let $\mathbf{V}$ be the closure in $\mathbf{R}^{3}$ of the convex hull of the graph $\mathscr{F}$ of $f_{\tau}(s)$ (recall the definition of $f(s)$ in Sec. 2 and that of $f_{\tau}$ in Sec. 3):

$$
\mathbf{V}=\overline{\operatorname{co}}\left\{(\mathbf{y}, z) \mid \mathbf{y}(s) \in \partial \Omega_{\tau}, \quad z=f_{\tau}(s), s \in[0, L)\right\} .
$$

For $\mathbf{x} \in \operatorname{co} \bar{\Omega}_{\tau}$ arbitrarily fixed, consider the set

$$
\Sigma(\mathbf{x}) \equiv\{z \in \mathbf{R} \mid \mathbf{x}+z \mathbf{e} \in \mathbf{V}\},
$$

and define

$$
\psi_{\tau}^{+}(\mathbf{x}) \equiv \sup _{z \in \mathbf{R}} \Sigma(\mathbf{x}), \quad \psi_{\tau}^{-}(\mathbf{x}) \equiv \inf _{z \in \mathbf{R}} \Sigma(\mathbf{x}),
$$

so that the following sets

$$
\begin{aligned}
(\partial \mathbf{V})^{+} & \equiv\left\{(\mathbf{x}, z) \mid \mathbf{x} \in \operatorname{co} \bar{\Omega}_{\tau}, z=\psi_{\tau}^{+}(\mathbf{x})\right\}, \\
(\partial \mathbf{V})^{-} & \equiv\left\{(\mathbf{x}, z) \mid \mathbf{x} \in \operatorname{co} \bar{\Omega}_{\tau}, z=\psi_{\tau}^{-}(\mathbf{x})\right\}
\end{aligned}
$$

are, respectively, the graphs of the upper and lower parts of $\partial \mathbf{V}$. 
Proposition 2. Under the hypotheses of the Main Theorem, we have $\mathscr{F} \subset(\partial \mathbf{V})^{+}$. Proof. Let $\left(\mathbf{y}, z^{\prime}\right) \equiv \mathbf{X}^{\prime} \in \mathscr{F}$ be arbitrarily fixed and $\mathbf{X} \equiv(\mathbf{y}, z) \in \mathbf{V}$.

We recall that

$$
\begin{aligned}
& \mathbf{V}=\left\{\mathbf{X} \in \mathbf{R}^{3} \mid \mathbf{X}=\mathbf{Y}_{0}+\alpha\left(\mathbf{Y}_{1}-\mathbf{Y}_{0}\right)+\beta\left(\mathbf{Y}_{2}-\mathbf{Y}_{0}\right)\right. \\
&\text { with } \left.\mathbf{Y}_{0}, \mathbf{Y}_{1}, \mathbf{Y}_{2} \in \mathscr{F}, \alpha, \beta \geq 0, \text { and } \alpha+\beta \leq 1\right\} .
\end{aligned}
$$

Since $\mathbf{X}^{\prime}$ and $\mathbf{Y}_{i}$ belong to $\mathscr{F}$ they can be expressed in the form

$$
\mathbf{X}^{\prime} \equiv\left(\mathbf{y}\left(s^{\prime}\right), f_{\tau}\left(s^{\prime}\right)\right), \quad \mathbf{Y}_{i} \equiv\left(\mathbf{y}\left(s_{i}\right), f_{\tau}\left(s_{i}\right)\right) \quad(i=1,2),
$$

and, because of (B1) and (B2), we have

(i) $\mathbf{v}_{\tau}\left(s^{\prime}\right) \cdot\left(\mathbf{y}\left(s_{0}\right)-\mathbf{y}\left(s^{\prime}\right)\right) \geq f_{\tau}\left(s_{0}\right)-f_{\tau}\left(s^{\prime}\right)$,

(ii) $\mathbf{v}_{\tau}\left(s^{\prime}\right) \cdot\left(\mathbf{y}\left(s_{1}\right)-\mathbf{y}\left(s^{\prime}\right)\right) \geq f_{\tau}\left(s_{1}\right)-f_{\tau}\left(s^{\prime}\right)$,

(iii) $\mathbf{v}_{\tau}\left(s^{\prime}\right) \cdot\left(\mathbf{y}\left(s_{2}\right)-\mathbf{y}\left(s^{\prime}\right)\right) \geq f_{\tau}\left(s_{2}\right)-f_{\tau}\left(s^{\prime}\right)$.

Since $\alpha, \beta>0$ we could combine (i), (ii), (iii) the following way:

$$
\text { (i) }+\alpha((\text { ii })-(\text { i }))+\beta((\text { iii })-(\mathrm{i})) \text {. }
$$

This would lead to

$$
\begin{aligned}
\mathbf{v}_{\tau}\left(s^{\prime}\right) \cdot\left[\left(\mathbf{y}\left(s_{0}\right)-\mathbf{y}\left(s^{\prime}\right)\right)+\alpha\left(\mathbf{y}\left(s_{1}\right)-\mathbf{y}\left(s_{0}\right)\right)+\beta\left(\mathbf{y}\left(s_{2}\right)-\mathbf{y}\left(s_{0}\right)\right)\right] \\
\\
\geq-f_{\tau}\left(s^{\prime}\right)+f_{\tau}\left(s_{0}\right)+\alpha\left(f_{\tau}\left(s_{1}\right)-f_{\tau}\left(s_{0}\right)\right)+\beta\left(f_{\tau}\left(s_{2}\right)-f_{\tau}\left(s_{0}\right)\right),
\end{aligned}
$$

that is,

$$
\mathbf{v}_{\tau} \cdot\left(\mathbf{y}-\mathbf{y}^{\prime}\right) \geq z-z^{\prime}
$$

Therefore, if $\mathbf{y}=\mathbf{y}^{\prime}$ it follows that $z^{\prime} \geq z$, that is, $\mathbf{X}^{\prime} \in \partial \mathbf{V}^{+}$.

Observe that, if $\mathbf{X} \in \partial \mathbf{V}^{+}$, the last inequality can be rewritten in the form

$$
\mathbf{v}_{\tau} \cdot\left(\mathbf{y}-\mathbf{y}^{\prime}\right)+f_{\tau} \geq \psi_{\tau}^{+} .
$$

ReMark 2. The proof of Proposition 2 is manifestly simplified by the use of the gradient $\mathbf{v}_{\tau}(s)$ instead of the normal derivative of $g_{\tau}(s)$. This justifies, a posteriori, the choice we made by writing the boundary conditions in the form (9) and (10).

Let us now define the following surface over $\Omega$ :

$$
z=\tilde{\phi}(\mathbf{x})= \begin{cases}\phi_{\tau}(\mathbf{x}) & \text { if } \mathbf{x} \in \mathscr{S}_{\tau}, \\ \psi_{\tau}^{+}(\mathbf{x}) & \text { if } \mathbf{x} \in \Omega-\mathscr{S}_{\tau},\end{cases}
$$

$z=\phi_{\tau}(\mathbf{x})$ being the Airy function corresponding to $\mathbf{T}_{\tau}$ over $\mathscr{S}_{\tau}$.

Proposition 2 implies $\psi_{\tau \mid \partial \Omega_{\tau}}^{+}=f_{\tau}$, that is, the continuity of $z=\tilde{\phi}(\mathbf{x})$ on $\partial \Omega_{\tau}$. Moreover, not only the functions $z=\phi_{\tau}(\mathbf{x})$ and $z=\psi_{\tau}^{+}(\mathbf{x})$ are concave in their domains of definition, but also $z=\tilde{\phi}(\mathbf{x})$ is concave over $\Omega$. Indeed, since the Hessian matrix of $\phi_{\tau}$ is negative semidefinite, if $\mathbf{x} \in \mathscr{S}_{\tau}, \mathbf{y} \in \Omega_{\tau}$, and $(1-\lambda) \mathbf{x}+\lambda \mathbf{y} \in$ $\mathscr{S}_{\tau}$ for $\lambda \in\left[0, \lambda^{0}\right],(1-\lambda) \mathbf{x}+\lambda \mathbf{y} \in \Omega_{\tau}$ for $\lambda \in\left[\lambda^{0}, 1\right]$, then

$$
\mathbf{v}_{\tau}^{0} \cdot\left(\mathbf{x}-\mathbf{y}^{0}\right)+f_{\tau}^{0} \geq \phi_{\tau}(\mathbf{x}) \text {, }
$$




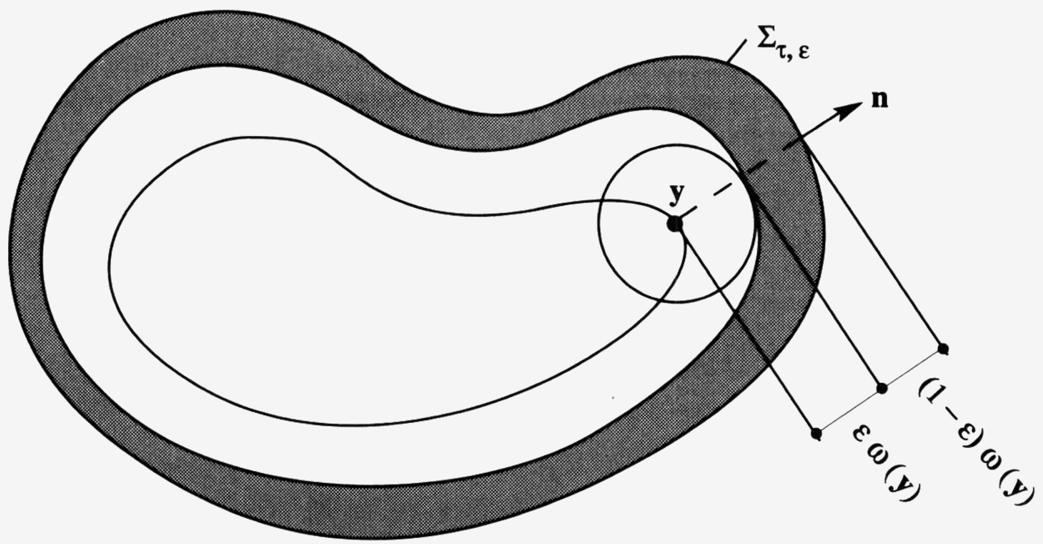

FIG. 4.

whilst, from $(28)$

$$
\mathbf{v}_{\tau}^{0} \cdot\left(\mathbf{y}-\mathbf{y}^{0}\right)+f_{\tau}^{0} \geq \psi_{\tau}^{+}(\mathbf{y}) .
$$

Combining these two inequalities and putting $\phi^{0}=\phi\left(\mathbf{y}^{0}\right)$ one easily obtains

$$
\phi^{0} \geq\left(1-\lambda^{0}\right) \phi(\mathbf{x})+\lambda^{0} \phi(\mathbf{y}) .
$$

Since $\phi_{\tau}$ and $\psi_{\tau}^{+}$are (locally) concave in their domains then

$$
\begin{gathered}
\phi\left[\frac{\lambda^{0}-\lambda}{\lambda^{0}} \mathbf{x}+\frac{\lambda}{\lambda^{0}} \mathbf{y}^{0}\right] \geq \frac{\lambda^{0}-\lambda}{\lambda^{0}} \phi(\mathbf{x})+\frac{\lambda}{\lambda_{0}} \phi^{0}, \quad \lambda \in\left[0, \lambda^{0}\right], \\
\phi\left[\frac{1-\lambda}{1-\lambda^{0}} \mathbf{y}^{0}+\frac{\lambda-\lambda^{0}}{1-\lambda^{0}} \mathbf{y}\right] \geq \frac{1-\lambda}{1-\lambda^{0}} \phi^{0}+\frac{\lambda-\lambda^{0}}{1-\lambda_{0}} \phi(\mathbf{y}), \quad \lambda \in\left[\lambda^{0}, 1\right] ;
\end{gathered}
$$

therefore,

$$
\begin{gathered}
\phi\left[\frac{\lambda^{0}-\lambda}{\lambda^{0}} \mathbf{x}+\frac{\lambda}{\lambda^{0}} \mathbf{y}^{0}\right] \geq \frac{\lambda^{0}-\lambda}{\lambda^{0}} \phi(\mathbf{x})+\frac{\lambda}{\lambda_{0}}\left[\left(1-\lambda^{0}\right) \phi(\mathbf{x})+\lambda^{0} \phi(\mathbf{y})\right], \quad \lambda \in\left[0, \lambda^{0}\right], \\
\phi\left[\frac{1-\lambda}{1-\lambda^{0}} \mathbf{y}^{0}+\frac{\lambda-\lambda^{0}}{1-\lambda^{0}} \mathbf{y}\right] \geq \frac{1-\lambda}{1-\lambda^{0}}\left[\left(1-\lambda^{0}\right) \phi(\mathbf{x})+\lambda^{0} \phi(\mathbf{y})\right]+\frac{\lambda-\lambda^{0}}{1-\lambda_{0}} \phi(\mathbf{y}), \quad \lambda \in\left[\lambda^{0}, 1\right] .
\end{gathered}
$$

These two inequalities, after some algebra, reduce to the form

$$
\phi[(1-\lambda) \mathbf{x}+\lambda \mathbf{y}] \geq(1-\lambda) \phi(\mathbf{x})+\lambda \phi(\mathbf{y}), \quad \lambda \in[0,1] .
$$

However, $z \in \tilde{\phi}(\mathbf{x})$ need not be smooth.

3.f. Regularization of $z=\tilde{\phi}(\mathbf{x})$. The function $z=\tilde{\phi}(\mathbf{x})$ verifies the boundary conditions (8) and (9) on $\partial \Omega$. However, since it is $C^{3}$ on $\mathscr{S}_{\tau}$ but only locally Lipschitz continuous over $\Omega$ we need to apply some smoothing argument.

To simplify notation let us set

$$
\omega(\mathbf{u})=\operatorname{dist}(\mathbf{u}, \partial \Omega), \quad \mathbf{u} \in \Omega,
$$

and, for an arbitrary $\varepsilon \in(0,1)$, let us put

$$
\Sigma_{\tau, \varepsilon}=\left\{\mathbf{u} \in \mathscr{S}_{\tau} \mid \mathbf{u}=\mathbf{y}-\lambda \omega(\mathbf{y}) \nabla \omega, \quad \lambda \in(\varepsilon, 1), \mathbf{y} \in \partial \Omega_{\tau}\right\} .
$$


Let now $\chi(\mathbf{x})$ be a nonnegative symmetric mollifier and $B$ the unit ball centered at the origin in $\mathbf{R}^{2}$. Then let us consider the convolution of $\tilde{\phi}$ with $\chi$ on $B$, i.e.,

$$
\phi(\mathbf{x})=\int_{B} \chi\left(\mathbf{x}^{\prime}\right) \tilde{\phi}\left(\mathbf{x}+\varepsilon \mathbf{x}^{\prime} \rho(\mathbf{x})\right) d \mathbf{x}^{\prime}, \quad \mathbf{x} \in \Omega,
$$

where $\rho$ is a smooth function such that

$$
\begin{gathered}
0<\rho(\mathbf{x}) \leq \omega(\mathbf{x}), \quad \mathbf{x} \in \Omega, \\
\rho(\mathbf{x})=\omega(\mathbf{x}), \quad \text { in } \Sigma_{\tau, \varepsilon} .
\end{gathered}
$$

The function $\phi$ is smooth in $\Sigma_{\tau, \varepsilon}$. This follows directly from (29) whereas, in $\Omega_{\tau, \varepsilon} \equiv \Omega-\Sigma_{\tau, \varepsilon}$, it is a consequence of the following alternative form of (29):

$$
\phi(\mathbf{x})=\frac{1}{\varepsilon^{2} \rho^{2}(\mathbf{x})} \int_{\Omega_{\tau, \varepsilon}} \chi\left(\frac{\mathbf{u}-\mathbf{x}}{\varepsilon \rho(\mathbf{x})}\right) \tilde{\phi}(\mathbf{u}) d \mathbf{u} .
$$

To show that $\phi$ is concave on $\Omega$, suppose first that $\tilde{\phi} \in C^{2}(\bar{\Omega})$. Then we have

$$
\begin{aligned}
\phi_{, i j}=\int_{B} \chi\left(\mathbf{x}^{\prime}\right)\left\{\tilde{\phi}_{, h k}\left(\mathbf{x}-\varepsilon \rho \mathbf{x}^{\prime}\right)\left(\delta_{i k}+\varepsilon \mathbf{x}_{k}^{\prime} \rho_{, i}\right)\left(\delta_{j h}+\varepsilon \mathbf{x}_{h}^{\prime} \rho_{, j}\right)\right. & \\
& \left.+\tilde{\phi}_{, h}\left(\mathbf{x}+\varepsilon \rho \mathbf{x}^{\prime}\right) \varepsilon \mathbf{x}_{h}^{\prime} \rho_{, i j}\right\} d \mathbf{x}^{\prime} .
\end{aligned}
$$

Since $\tilde{\phi}$ is concave, we have $\tilde{\phi}_{, i j} \zeta_{i} \zeta_{j} \leq 0$ for arbitrary $\zeta=\left(\zeta_{1}, \zeta_{2}\right) \in \mathbf{R}^{2}$; therefore,

$$
\phi_{, i j}(\mathbf{x}) \zeta_{i} \zeta_{j} \leq \int_{B} \chi\left(\mathbf{x}^{\prime}\right)\left\{\varepsilon \sup |D \tilde{\phi}| \sup \left|D^{2} \rho\right||\zeta|^{2}\right\} d \mathbf{x}^{\prime} \leq \varepsilon M .
$$

Since $\varepsilon>0$ is sufficiently small but otherwise arbitrary, we conclude that

$$
\phi_{, i j}(\mathbf{x}) \zeta_{i} \zeta_{j} \leq 0 \text {. }
$$

If $\tilde{\phi}$ were not $C^{2}$, we could approximate $\tilde{\phi}$ by smooth concave functions $\tilde{\phi}_{k}$. For a well-known property of mollifiers, the corresponding functions $\phi_{k}$ satisfy (30) and converge to $\phi$. This completes the proof of the main theorem.

Appendix I. Proof of (18) and of Lemma 1.

Proof of (18). Since $\nu$ is a unit vector we have $\nu \cdot d \nu / d s=0$, and therefore

$$
\frac{d \nu}{d s}=\lambda \boldsymbol{\sigma}
$$

with $\boldsymbol{\sigma}=\mathbf{e} \times \boldsymbol{\nu} ; \lambda$ measures the "bending" of $\boldsymbol{\nu}$ along $\partial \boldsymbol{\Omega}$. We want to determine $\lambda$ in terms of $\mathscr{R}(s)$ (see Sec. 3.a for the definition of $\mathscr{R}$; see also Fig. 2): by choosing $\mathscr{R} \nu$ as the position vector for the points of $\partial \Omega$ we have

$$
\frac{d(\mathscr{R} \boldsymbol{\nu})}{d s}=\mathbf{t} ;
$$

then

$$
\mathbf{t}=\mathscr{R} \frac{d \boldsymbol{\nu}}{d s}+\boldsymbol{\nu} \frac{d \mathscr{R}}{d s}=\lambda \mathscr{R} \boldsymbol{\sigma}+\boldsymbol{\nu} \frac{d \mathscr{R}}{d s} .
$$

Taking the scalar product first by $\mathbf{n}$ and then by $\nu$, we obtain

$$
0=\lambda \mathscr{R}(\boldsymbol{\sigma} \cdot \mathbf{n})+\frac{d \mathscr{R}}{d s}(\boldsymbol{\nu} \cdot \mathbf{n}), \quad \mathbf{t} \cdot \boldsymbol{\nu}=\frac{d \mathscr{R}}{d s},
$$


and, since (recall (17)) $\boldsymbol{\nu} \cdot \mathbf{n}=-\cos \alpha$ and $\boldsymbol{\sigma} \cdot \mathbf{n}=t \cdot \boldsymbol{\nu}=\sin \alpha$, we finally get

$$
\mathscr{R}=\frac{\cos \alpha}{\lambda} \text {. }
$$

Proof of Lemma 1. The natural basis vectors with respect to the curvilinear system $\left(\xi^{1}, \xi^{2}\right)$, defined through $(16)$, are

$$
\left\{\begin{array}{l}
\mathbf{a}_{1}=\frac{\partial \mathbf{x}}{\partial \xi^{1}}=\mathbf{t}(s)+\xi^{2} \frac{d \nu}{d s}(s)=\mathbf{t}(s)+\xi^{2} \frac{\cos \alpha}{\mathscr{R}} \boldsymbol{\sigma}(s), \\
\mathbf{a}_{2}=\frac{\partial \mathbf{x}}{\partial \xi^{2}}=\nu(s)
\end{array}\right.
$$

from which the contravariant components of the metric tensor $\mathbf{g}$ are easily computed. To this purpose let $\tilde{\alpha}\left(\xi^{1}, \xi^{2}\right)$ be the angle between $\mathbf{a}_{2}$ and its dual correspondent $\mathbf{a}^{2}$; of course, $\tilde{\alpha}\left(\xi^{1}, 0\right)=\alpha(s)=\alpha\left(\xi_{1}\right)$. Then notice that (32) yields

$$
\mathbf{a}_{1} \cdot \mathbf{a}_{2}=\sin \alpha
$$

on the other hand, we have

$$
\begin{gathered}
g_{12}=\mathbf{a}_{1} \cdot \mathbf{a}_{2}=\left|\mathbf{a}_{1}\right|\left|\mathbf{a}_{2}\right| \sin \tilde{\alpha} \\
g_{11}=\left|\mathbf{a}_{1}\right|^{2}=\sin ^{2} \alpha+\frac{\cos ^{2} \alpha}{\mathscr{R}}\left(\mathscr{R}-\xi^{2}\right)^{2}, \\
g_{22}=\left|\mathbf{a}_{2}\right|^{2}=1 ;
\end{gathered}
$$

(33) and (34) imply

$$
\tan \tilde{\alpha}\left(\xi^{1}, \xi^{2}\right)=\left(\tan \alpha\left(\xi^{1}\right)\right) \frac{\mathscr{R}\left(\xi^{1}\right)}{\mathscr{R}\left(\xi^{1}\right)-\xi^{2}} .
$$

By differentiating (35) we have

$$
\frac{\partial \tilde{\alpha}}{\partial \xi^{2}}=\frac{\sin ^{2} \tilde{\alpha}}{\mathscr{R} \tan \alpha} .
$$

The components of the metric tensor are therefore expressed in terms of $\alpha$ and $\tilde{\alpha}$ by

$$
\left(g_{i j}\right)=\left[\begin{array}{cc}
\frac{\sin ^{2} \alpha}{\sin ^{2} \dot{\alpha}} & \sin \alpha \\
\sin \alpha & 1
\end{array}\right] \text {. }
$$

To prove Lemma 1 we need to verify that the stress field $\mathbf{T}_{\tau}$ given by (21) is statically admissible, that is, satisfies (1) and (2).

Using the local coordinates defined by (17), condition (1), can be rewritten as

$$
\left\{\begin{array}{l}
T_{/ 1}^{11}+T_{/ 2}^{21}=0 \\
T_{/ 1}^{12}+T_{/ 2}^{22}=0
\end{array}\right.
$$

where "/" means the covariant derivative with respect to $\left(\xi^{1}, \xi^{2}\right)$.

The Christoffel symbols we need to make (36) explicit are easily computed: we have

$$
\left\{\begin{array}{l}
\Gamma_{22}^{1}=\Gamma_{22}^{2}=0 \\
\Gamma_{12}^{1}=-\frac{1}{\not h-\xi^{2}}
\end{array}\right.
$$


Inserting the stress field (21) $\mathbf{T}_{\tau}$ into (37) we get

$$
\begin{aligned}
\Gamma_{22}^{1} T_{\tau}^{22} & =0, \\
T_{\tau, 2}^{22}+\Gamma_{12}^{1} T_{\tau}^{22}+2 \Gamma_{22}^{2} T_{\tau}^{22} & =0,
\end{aligned}
$$

which, because of (38), turn out to be identically verified.

As far as the boundary condition is concerned, we have

$$
\mathbf{T}_{\tau} \cdot \mathbf{n}=-\mathbf{p}(s)(\boldsymbol{\nu} \cdot \mathbf{n}) \frac{1}{\cos \alpha}=\mathbf{p} .
$$

Appendix II. The case of a partially unloaded boundary. In the proof of the Main Theorem the hypothesis (ii) implies $\Gamma_{0}=\varnothing$ and plays a crucial role. However, let us notice that since we allow $\mathbf{R}\left(\mathbf{x}_{1}, \mathbf{x}_{2}\right)=\mathbf{M}\left(\mathbf{x}_{1}, \mathbf{x}_{2}\right)=0$ over a section $\operatorname{seg}\left(\mathbf{x}_{1}, \mathbf{x}_{2}\right)$ of $\Omega$, the existence of a s.a.f. $T$ over $\Omega$ trivially guarantees the existence of two s.a.f.'s $\mathbf{T}_{1}$ and $\mathbf{T}_{2}$ over the two parts $\mathscr{P}^{\prime}$ and $\mathscr{P}^{\prime \prime}$ of $\Omega$ lying on each side of $\operatorname{seg}\left(\mathbf{x}_{1}, \mathbf{x}_{2}\right)$. Obviously $\mathbf{p}$ vanishes over $\operatorname{seg}\left(\mathbf{x}_{1}, \mathbf{x}_{2}\right)$, which is a portion of the boundary of both $\mathscr{P}^{\prime}$ and $\mathscr{P}^{\prime \prime}$ (see Fig. 5). Let us notice that, in general, $\mathbf{p}$ is not even $C^{1}$ on the boundary of the body and the boundary itself is not $C^{1}$.

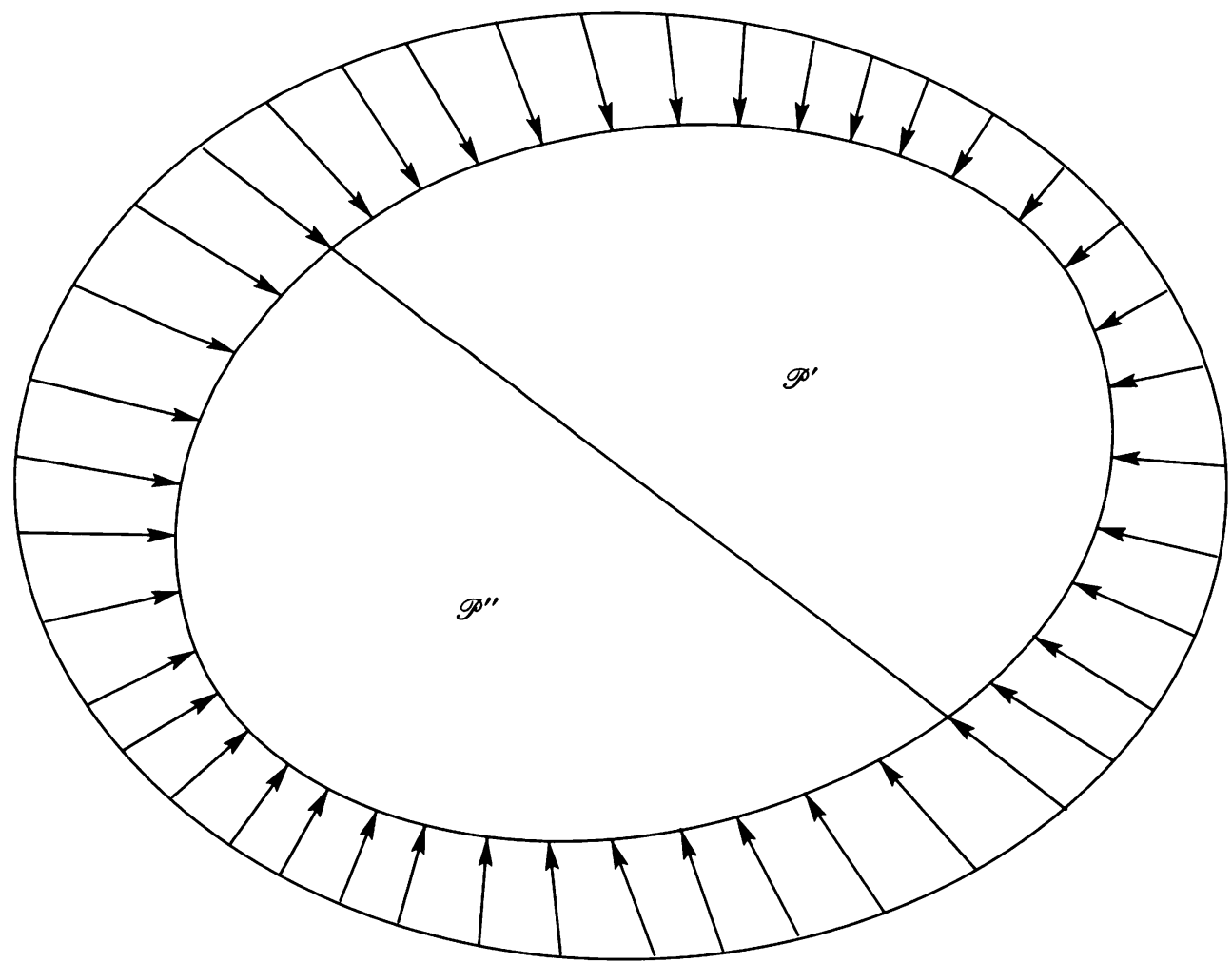

FIG. 5. 


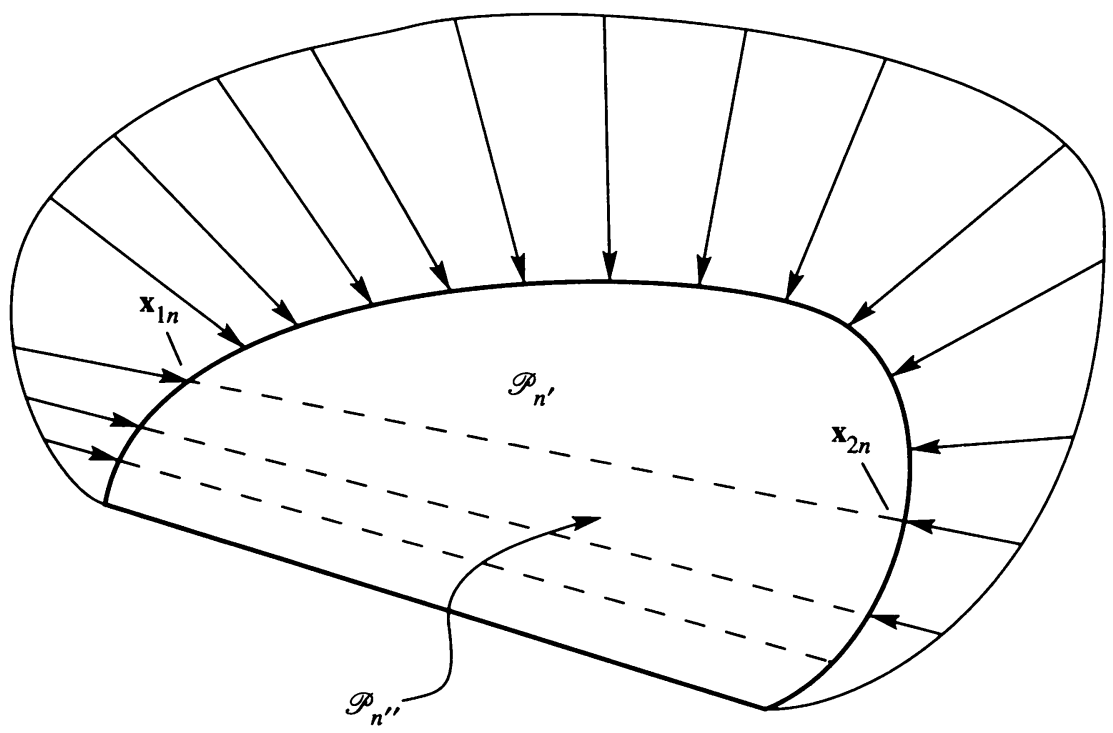

FIG. 6 .

Consider now the case shown in Fig. 6: the boundary of the body is not $C^{1}$ as before, but $\mathbf{p}$ is now a $C^{1}$ field over this boundary, since it goes to zero at the endpoints, $\mathbf{x}_{1}$ and $\mathbf{x}_{2}$, of the straight portion of the boundary. Let us also assume that $\operatorname{seg}\left(\mathbf{x}_{1}, \mathbf{x}_{2}\right)$ is the limit element of a congruence of unloaded segments and let $\operatorname{seg}\left(\mathbf{x}_{1 n}, \mathbf{x}_{2 n}\right)$ be a sequence selected from this congruence. For any finite $n$, consider the two parts $\mathscr{P}_{n^{\prime}}$ and $\mathscr{P}_{n^{\prime \prime}}$ shown in Fig. 6; because of the previous consideration we have a s.a.f. $\mathbf{T}_{n^{\prime}}$ over $\mathscr{P}_{n^{\prime}}$. The tensor field (21) provides a s.a.f. $\mathbf{T}_{n^{\prime \prime}}$ over the entire strip $\mathscr{P}_{n^{\prime \prime}}$.

The field

$$
T= \begin{cases}\mathbf{T}_{n^{\prime}}, & \mathbf{x} \in \mathscr{P}_{n^{\prime}}, \\ \mathbf{T}_{n^{\prime \prime}}, & \mathbf{x} \in \mathscr{P}_{n^{\prime \prime}}\end{cases}
$$

is continuous and provides a s.a.f. over the region $\Omega$ occupied by the body.

Other cases-like, for example, that shown in Fig. 7, where $\left(\mathbf{x}_{1}, \mathbf{x}_{2}\right) \in \mathscr{\mathscr { C }}$, $\operatorname{seg}\left(\mathbf{x}_{1}, \mathbf{x}_{2}\right)$ is unloaded and $\mathbf{p}$ goes smoothly to zero at the endpoints along $\partial \mathscr{P}^{\text {in }}$ while $\partial \mathscr{P}^{\prime \prime}$ is unloaded-seem to require more sophisticated techniques and a deeper understanding. We are actually unable to show the existence of a solution in this case.

At least in the first two cases discussed above we can say that the existence of a s.a.f. $T$ is guaranteed whenever $\mathscr{H}_{1} \subseteq \mathscr{H}_{\text {in }}$. In particular, if $\Omega$ is strictly convex, that is, the curvature of $\partial \Omega$ is uniformly bounded from below by a positive constant, $\mathscr{H}=\mathscr{H}$ and therefore the sole hypotheses (i) and (ii) of the Main Theorem become necessary and sufficient to the existence of a s.a.f. on $\Omega$ regardless of whether $\mathbf{p}=0$ or not on portions of $\partial \Omega$. 


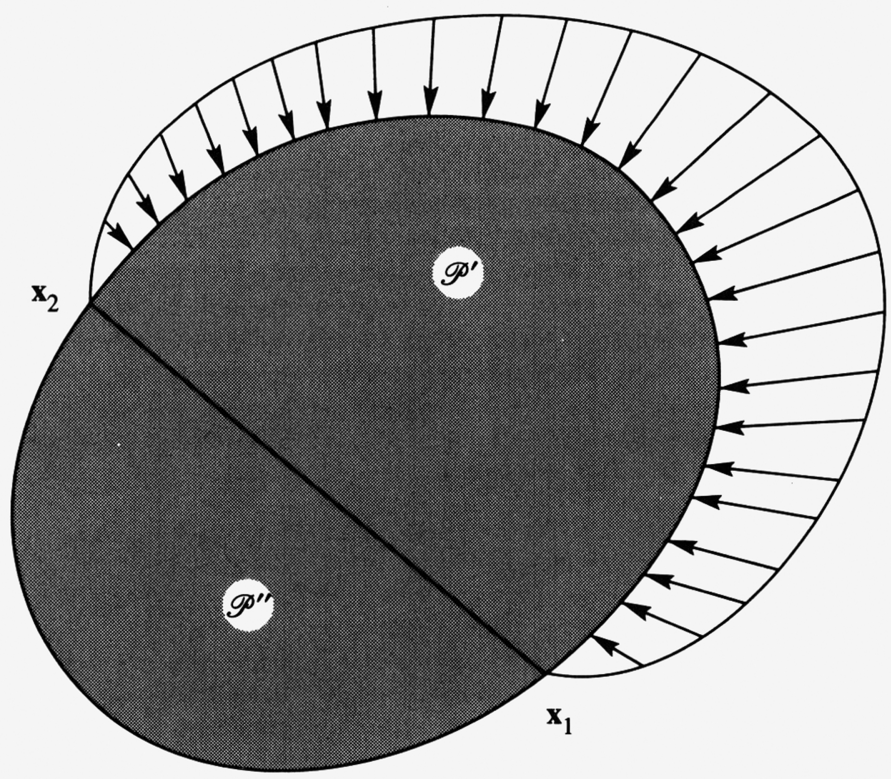

FIG. 7.

Acknowledgments. We wish to express our deep gratitude to one of the referees for his careful reading of this paper. Without his valuable comments and criticisms this work would probably have never reached this final form.

\section{REFERENCES}

[1] G. Del Piero, Recent developments in the mechanics of materials which do not support tension, Proc. Internat. Colloquium on Free Boundary Problems, Irsee, 1987. Research Notes in Mathematics, vol. 185, Pitman, 1990, pp. 2-24

[2] G. Del Piero, Constitutive equations and compatibility of the external loads for linear elastic masonry-like materials, Meccanica 24, 3 (1989)

[3] M. Giaquinta and E. Giusti, Researches on the equilibrium of masonry structures, Arch. Rational Mech. Anal. 88, 359-392 (1986)

[4] M. E. Gurtin, The linear theory of elasticity, Handbuch der Physik, band VIa/2, 1972

[5] G. Romano and E. Sacco, Sul calcolo di strutture non reagenti a trazione, Atti VII Congresso Aimeta, Trieste, 1984 